\title{
Melt Pool Features Analysis Using a High Speed Coaxial Monitoring System for Laser Powder Bed Fusion of Ti-6Al-4V Grade 23
}

Aditi Thanki ( $\square$ aditi.thanki@kuleuven.be )

KU Leuven

Louca Goossens

KU Leuven

Agusmian Partogi Ompusunggu

Flanders Make

Mohamad Bayat

Technical University of Denmark

Abdellatif Bey-Temsamani

Flanders Make

Brecht Van Hooreweder

KU Leuven

Jean-Pierre Kruth

KU Leuven

Ann Witvrouw

KU Leuven

\section{Research Article}

Keywords: Laser Powder Bed Fusion (LPBF), Melt pool monitoring, Spatter analysis, Keyhole porosity, Finite volume modelling, X-ray computed tomography (X-CT)

Posted Date: December 20th, 2021

DOI: https://doi.org/10.21203/rs.3.rs-1128009/v1

License: (c) (i) This work is licensed under a Creative Commons Attribution 4.0 International License.

Read Full License 


\section{Melt pool features analysis using a high speed coaxial monitoring system for laser powder bed fusion of Ti-6Al-4V grade 23}

Aditi Thanki ${ }^{1,2, *}$, Louca Goossens ${ }^{1,2, *}$, Agusmian Partogi Ompusunggu ${ }^{3}$, Mohamad Bayat ${ }^{4}$, Abdellatif Bey-Temsamani ${ }^{3}$, Brecht Van Hooreweder ${ }^{1,2}$, Jean-Pierre Kruth ${ }^{1,2}$, Ann Witvrouw ${ }^{1,2}$

${ }^{1}$ KU Leuven, Department of Mechanical Engineering, Celestijnenlaan 300B, 3001 Heverlee, Belgium

${ }^{2}$ Member of Flanders Make, Leuven, Belgium

${ }^{3}$ Flanders Make, Gaston Geenslaan 8, 3001 Heverlee, Belgium

${ }^{4}$ Technical University of Denmark, Department of Mechanical Engineering, building 425, Lyngby, Denmark

*Joint first authors.

E-mail address:

Aditi.Thanki@kuleuven.be (A. Thanki)

Louca.Goossens@kuleuven.be (L. R. Goossens)

Ann.Witvrouw@kuleuven.be (A. Witvrouw)

\section{Abstract}

In laser powder bed fusion (LPBF), defects such as pores or cracks can seriously affect the final part quality and lifetime. Keyhole porosity, being one type of porosity defects in LPBF, results from excessive energy density which may be due to changes in process parameters (laser power and scan speed) and/or result from the part's geometry and/or hatching strategies. To study the possible occurrence of keyhole pores, experimental work as well as simulations were carried out for optimum and high volumetric energy density conditions in Ti-6Al-4V grade 23. By decreasing the scanning speed from $1000 \mathrm{~mm} / \mathrm{s}$ to $500 \mathrm{~mm} / \mathrm{s}$ for a fixed laser power of $170 \mathrm{~W}$, keyhole porosities are formed and later observed by X-ray computed tomography. Melt pool images are recorded in real-time during the LPBF process by using a high speed coaxial NearInfrared (NIR) camera monitoring system. The recorded images are then pre-processed using a set of image processing steps to generate binary images. From the binary images, geometrical features of the melt pool and features that characterize the spatter particles formation and ejection from the melt pool are calculated. The experimental data clearly show spatter patterns in case of keyhole porosity formation at low scan speed. A correlation between the number of pores and the amount of spatter is observed. Besides the experimental work, a previously developed, high fidelity finite volume numerical model was used to simulate the melt pool dynamics with similar process parameters as in the experiment. Simulation results illustrate and confirm the keyhole porosity formation by decreasing laser scan speed.

Keywords: Laser Powder Bed Fusion (LPBF), Melt pool monitoring, Spatter analysis, Keyhole porosity, Finite volume modelling, X-ray computed tomography (X-CT) 


\section{Introduction}

Selective laser melting (SLM), otherwise called metal laser powder bed fusion (LPBF), is a process where a 3-D metal part is built in a layer-by-layer manner by utilizing a laser to melt the metal powder selectively in a powder bed. Kruth and der Schueren, 1995 [1] studied this technique in the mid 1990's, followed by Meiners, 1999 [2]. In this process, a thin layer of metallic powder is molten at selected places by scanning a laser beam over a powder bed as per a predefined scanning strategy (Figure 1). The main advantage of this process is that it enables the manufacturing of complex metallic parts. Applications of the LPBF process can be found in a variety of sectors. Examples of those are the medical sector [3], tool industries [4], automotive industry [5] and aerospace industry [6]. The present state-of-the-art LPBF process with optimized processing parameters normally leads to near or over $99 \%$ part densities. Nevertheless, local defects such as pores might exist. These imperfections can be a concern as they can cause variations in the mechanical properties of produced parts. The presence of these porosities becomes even more problematic when the defects are present in critical zones (e.g. subject to high mechanical stress) of the produced parts and thus limit the use of LPBF in critical applications, such as aerospace or the medical industry. These undesirable defects might appear due to non-idealities related to the processing parameter settings as well as hardware behaviour, such as scanning mirror acceleration/deceleration, leading to over-melting or insufficient melting.

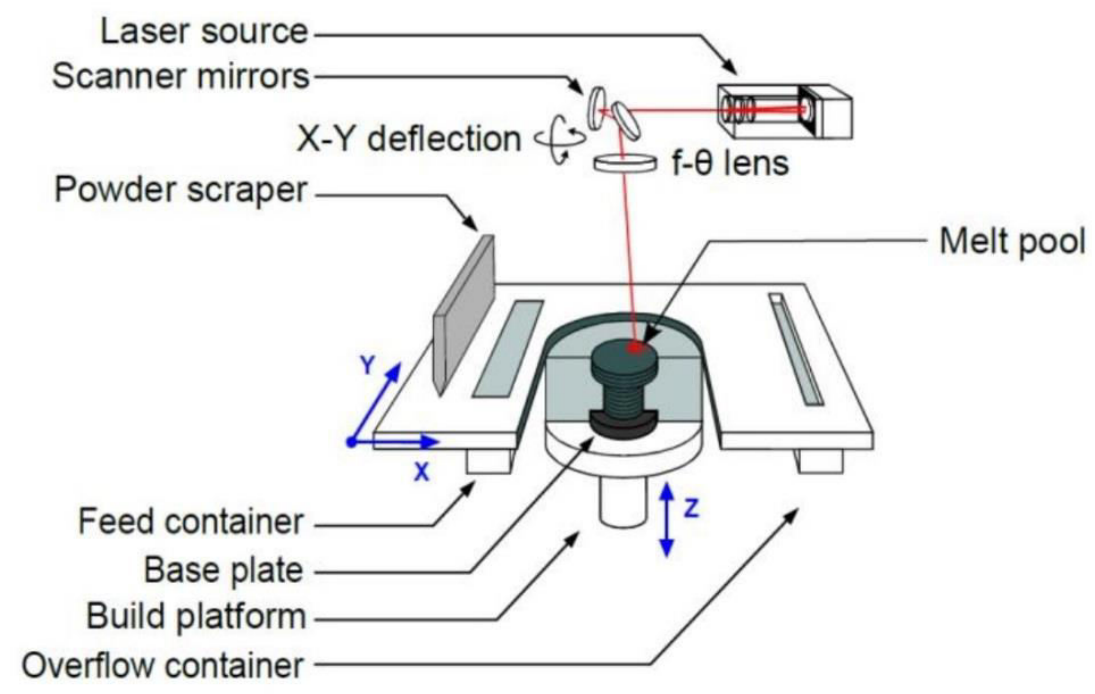

There are many reasons for pore formation in LPBF, such as lack of fusion [8], Plateau-Rayleigh instability [9], overheating in overhanging structures [10] or keyhole-induced pores [11]. The mechanisms behind the latter is the keyhole phenomenon, caused by high volumetric energy conditions, with the resulting porosity being denoted as a keyhole pore. During keyhole mode, the temperature at the centre of the laser beam and at the bottom part of the keyhole reaches values above the vaporization temperature of the metal. This prompts an exponential increase in the recoil pressure and a linear reduction in the surface tension, opening and stabilizing the keyhole void [12]. Nevertheless, when the keyhole is dragged along during scanning, instabilities 
can arise. Matsunawa et al., 1998 [13] showed that as a result of the keyhole front inclination angle, the evaporation site can shift to different locations on the front keyhole wall. This can perturb the rear melt pool wall and as a result generate instabilities and keyhole oscillation. At this moment, the laser rays lose the vast majority of their energy because of multiple collisions. This redistribution of the laser energy can significantly change the local temperature distribution inside of the keyhole and subsequently also the local recoil pressure. With the decrease of the temperature and recoil pressure, a local rise of the surface tension is observed. As a result, the balance of forces is disrupted and the keyhole can no longer be sustained locally, leading to a rapid collapse of the keyhole. Consequently, a gas pocket might be trapped due to the abrupt breakdown of the rear wall, forming what is called a keyhole-induced pore at the bottom of the keyhole.

Keyhole and keyhole-induced pore formation has already been studied by many authors. King et conduction to keyhole mode laser melting. They identified the conditions for keyhole formation through single-track experiments for 316L stainless steel. Martin et al., 2019b [14] elucidated the mechanism of keyhole pore formation during laser turn points using in situ X-ray imaging and multi-physics simulations. Khairallah et al., 2016 [9] developed a numerical model that included the recoil pressure and the Marangoni effect and investigated the melt pool evolution. Bayat et al., 2019 [12] developed a high-fidelity multiphysics numerical model taking into account multi physics phenomena during LPBF. They studied the formation of keyhole porosities in printed single tracks of Ti6Al4V and validated the simulation results with experimental results. Cunningham et al., 2019 [15] used an ultrahigh-speed synchrotron X-ray imaging to capture the keyhole evolution across a range of process conditions for a Ti6Al4V alloy.

The effect of the process parameters on the quality is indirect, namely through the melting process. Therefore, it is important to understand the relation between the melting behaviour and the part quality. It is known that the melt pool characteristics such as melt pool geometry and its thermal distribution are an indicator of the final microstructure, which can strongly affect the mechanical property of the final build part [16]. An in-process melt pool monitoring system is a useful tool to inspect the melt pool behaviour during the LPBF process [17]. Along with the insitu observations, multi-physics simulations of the LPBF process are very helpful in understanding the mechanisms of defect formation. These models enable the analysis of the melt pool's thermo-fluidic behaviour with a very fine spatial-temporal resolution and involve various physical phenomena, e.g. evaporation, solidification, capillarity, etc. Multi-physics models have already been implemented by some of the co-authors to study the mechanisms of lack-of-fusion and keyhole-induced porosities during the LPBF process [12]. These models are found to be very accurate in determining the size, shape and the possible locations of the pores.

During the powder bed fusion process, spatter particles may eject from the melt pool due to multi-physics phenomena. These spatter particles can have an impact on the part quality as large spatter particles that land on the top of the powder layer may attenuate the laser beam, resulting in lack of fusion porosity, and may increase surface roughness. Therefore, the identification and analysis of spatter are considered important. Matthews et al., 2016 [18] observed the denudation phenomena near the laser scan track using high-speed imaging and explained that for a typical environment, inward gas flow driven by a metal vapour jet due to the Bernoulli effect was the dominant driving force for denuding powder near a melt track. Ly et al., 2017 [19] explained the 
physics of droplet ejection by ultra-high speed imaging of the melt pool. They presented detailed experiments and finite element modelling of metal micro-droplets to explain the physics of the laser-vapour-driven particle entrainment. Gunenthiram et al., 2018 [20] investigated metallic ejections generated during SLM using a high speed camera. Image analysis was performed for spatter analysis and a correlation was proposed between the volume of spatters and volume energy densities (VED) to identify melt-pool stability regimes. Repossini et al., 2017 [21] monitored spatter using an off-axis camera with a large field-of-view installed on their machine. Basic image processing, like the simple thresholding method, was used to binarize the recorded images. The binary images are then further processed to extract some features related to spatter, like the number of spatters, the average spatter area and the speed of ejected spatters.

This paper focuses on melt pool and spatter analysis in LPBF builds for Ti-6Al-4V grade 23. Both the melt pool characteristics (intensity, geometry) and spatter can be observed with the inprocess coaxial monitoring system. The current work presents a first attempt to characterize spatter on coaxial camera images recorded from a coaxial monitoring system and to link it to the keyhole pores' formation. An important challenge in analysing the coaxial camera images is that in many cases the melt pool is overlapping with the spatters. In addition, in some cases, the intensity of spatters in the images is quite low. Hence, applying a basic thresholding method to the images will miss-detect spatters ejected from the melt pool. Therefore, a novel combination of image processing steps are proposed in this paper to highlight and enhance both spatters overlapping with the melt pool and spatters with low intensity. The paper is structured as follows. Section 2 reviews the experimental procedure including the description of the measurement set-up and the algorithmic flow to process the collected data and to transform them into monitoring features. Furthermore, the measurements obtained through optical microscopy and X-ray tomography, as well as melt pool CFD simulations are described. Section 3 discusses the results at nominal and deviating volumetric energy density conditions obtained from experimental and modelling analyses. Conclusions are formulated in Section 4.

\section{Experimental procedure}

\subsection{LPBF process}

An in-house developed LPBF machine of KU Leuven (LM-Q) was used to build the parts under investigation. This machine is equipped with an Ytterbium (Yb) continuous wave fibre laser source with a wavelength of $1080 \mathrm{~nm}$ and maximum output power of $1 \mathrm{~kW}$. The focused laser beam has a spot diameter of $37.5 \mu \mathrm{m}\left(\varnothing_{1 / e^{2}}: 13.5 \%\right.$ of maximum). The subject of the investigation presented here are single-line tracks, built on dense LPBF substrates (10 mm x 10 $\mathrm{mm} \times 5 \mathrm{~mm}$ ). The feedstock material used is Ti-6Al-4V grade 23 powder, with a powder particle size ranging between $15-45 \mu \mathrm{m}$. The dense substrates are built using optimized process parameters (see Table 1) that are, in their turn, optimized based on the maximum achieved Archimedes' part density. The layer thickness is set at $30 \mu \mathrm{m}$, both for the substrates and the single line tracks. The scan strategy for the substrate is contour first, with a bi-directional infill and a rotation of 90 degrees between layers. The single tracks are produced simultaneously with the substrates, in a single job, to mimic normal processing conditions. For the single line tracks, the Volumetric Energy Density (VED) is modified by adapting both the scan speed and the laser power. The applied VEDs are subdivided into three regions, identified as being low, optimum and high VED (Table 1). The VED in Table 1 is calculated by using the following equation: 


$$
V E D=\frac{P}{v \cdot d \cdot t}
$$

where VED is the applied volumetric energy density $\left(\mathrm{J} / \mathrm{mm}^{3}\right), P$ is the laser power $(\mathrm{W}), v$ is the scan speed $(\mathrm{mm} / \mathrm{s}), t$ is the layer thickness $(\mathrm{mm})$ and $d$ is the spot diameter $(\mathrm{mm})$.

Table 1. Process parameter for LPBF of Ti-6Al-4V.

\begin{tabular}{|c|c|c|c|c|}
\hline Condition & ID & $\begin{array}{c}\text { Laser } \\
\text { Power } / \mathbf{W}\end{array}$ & $\begin{array}{c}\text { Scan } \\
\text { Speed / } \\
\mathbf{m m} \cdot \mathbf{s}^{-\mathbf{1}}\end{array}$ & $\begin{array}{c}\text { Volumetric energy } \\
\text { density }\left(\text { VED) / J } \cdot \mathbf{m m}^{-3}\right.\end{array}$ \\
\hline \multirow{2}{*}{ Optimum VED } & 1 & 170 & 1000 & 151.11 \\
\hline \multirow{2}{*}{ High VED } & 2 & 340 & 1000 & \multirow{2}{*}{302.22} \\
\cline { 2 - 4 } & 3 & 170 & 500 & \multirow{2}{*}{30} \\
\hline \multirow{2}{*}{ Low VED } & 4 & 85 & 1000 & \multirow{2}{*}{75.55} \\
\cline { 2 - 4 } & 5 & 170 & 2000 & \multicolumn{2}{|c|}{} \\
\hline
\end{tabular}

In previous work [22], in which the same samples were examined, it was shown that for an identical high VED ${ }^{1}$ (i.e. ID\# 2 and 3), a lower scan speed at the optimum laser power resulted in keyhole porosities, while a higher laser power at the optimum scan speed did not have the same detrimental effect. This study focuses on the analysis of the experimental condition leading to keyhole porosities (ID\# 3 in Table 1). A comparison (both experimentally and through modelling) is thus made with the condition under optimum process parameters (ID\# 1 in Table 1) and the condition at high VED (ID\# 3 in Table 1).

Extracting melt pool dimensions using metallographic analysis is challenging for Ti-6Al-4V, as the melt pool boundaries are not readily revealed by etching. Alternatively, the melt pool width is measured by imaging the top surface, by means of a Keyence VHX-600 optical microscope. The depth dimension is estimated by examining the keyhole porosity depth using X-CT crosssections.

\subsection{In-process melt pool monitoring}

The in-house developed LPBF machine of KU Leuven (LM-Q) is equipped with a coaxial melt pool monitoring set-up. A description of the conceptual operation and components is given in this section. The general layout is schematically shown in Figure 2. The laser source of $1080 \mathrm{~nm}$ wavelength is collimated (d), coupled into free space, and guided through an optic with a thinfilm interference filter (c). The filter is designed to have a cut-on wavelength of $950 \mathrm{~nm}$ i.e.

\footnotetext{
${ }^{1}$ In [22] the laser spot diameter was assumed to be $50 \mu \mathrm{m}$, resulting in slightly smaller reported VED values as compared to the values reported here. Afterwards the laser spot size was measured to be $37,5 \mu \mathrm{m}$ leading to the corrected VED values shown in Table 1.
} 
wavelengths above $950 \mathrm{~nm}$ are transmitted, while those below the cut-on wavelength are reflected. This filter is thus allowing the laser beam to pass freely through the optic. After the filter, the laser is positioned and focused on the powder bed using a Galvano-scanner and f-theta lens combination (b). When the laser hits the powder at the workplace (a), a melt pool is created and according to the black-body radiation theory (Planck's law), a spectrum of radiation is emitted depending on the object temperature. Part of the generated melt pool radiation or process light is transmitted back into the f-theta lens and Galvano-scanner combination (b). The process light is then separated from the laser source by reflecting off the thin-film interference filter (c). After separation, the process light is distributed evenly among the two sensors using a beam splitter (e). The two sensors are a silicon photodiode (f) [23] and a CMOS camera (g) [24]. Both the sensors are band-passed filtered to have the near-infrared (NIR) wavelength range between 800-950 $\mathrm{nm}$ for observation of the melt pool radiation. Filtering eliminates any remaining laser emission, as well as prohibits any stray ambient light from interfering with the measurements.

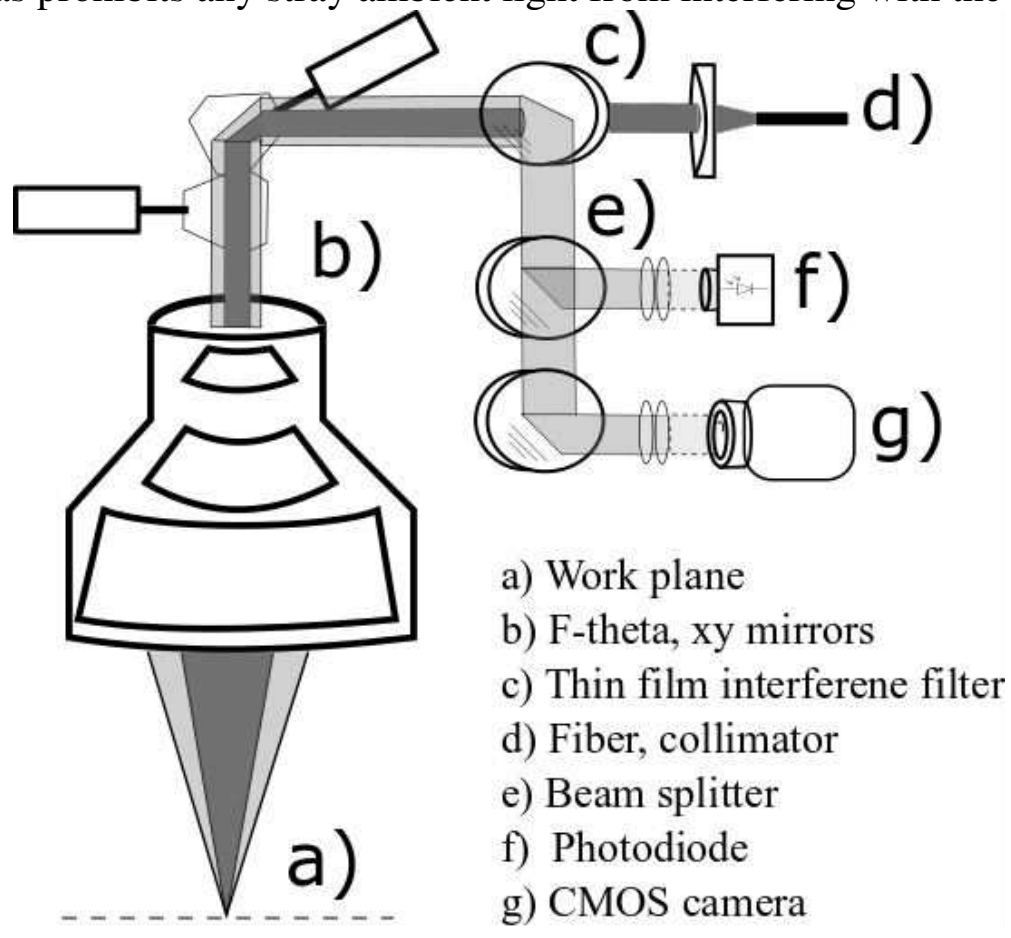

Figure 2. Schematic overview of the monitoring setup.

The analogue signal originating from the photodiode (f) is sampled using a sample and hold analogue-to-digital converter with 16-bit resolution $(-10,10 \mathrm{~V})$ with the sampling frequency of $20 \mathrm{kHz}$. The photodiode signal is synchronized and simultaneously logged together with the scanner XY-positions.

The CMOS camera $(\mathrm{g})$ is connected to a separate frame grabber system, using the Camera Link® interface. The images measure 80 by 80 pixels and have an 8 -bit grayscale pixel depth. The pixel size is measured to be $14 \mu \mathrm{m}$ by employing an R3L3S3P concentric square target calibration plate manufactured by Thorlabs and is placed in the working plane. This results in a field of view of $1120 \mu \mathrm{m}$ by $1120 \mu \mathrm{m}$. Thus, both the melt pool and surrounding environment are captured by the images, allowing for the occasional spatter to be captured by the camera. An example image 
during operation is shown in Figure 4, showing both the melt pool and some spatter particles being ejected from said melt pool. The grayscale image intensities are inverted for clarity ( $0=$ white, $255=$ black $)$.

The acquisition frame rate is fixed at 20,000 fps (frames per second). The camera acquisition is triggered with the machine controller's data logging signal, synchronizing photodiode, scanner coordinates and camera images. All images are transferred and stored to a parallel RAID 0 SSD array containing 6 disks, capable of sustaining a data rate exceeding $1 \mathrm{~GB} / \mathrm{s}$. This assures that the maximum supported Camera Link ${ }^{\circledR}$ data rate of $850 \mathrm{MB} / \mathrm{s}$ is achievable throughout the entire process.

\subsection{Image processing steps for extracting geometrical and spatter features from the camera melt pool images}

Figure 3 shows the flowchart of the image processing and features extraction steps for characterising the melt pool geometry and spatters applied to the coaxial camera image. The innovation proposed in this paper is a combination of different existing image processing algorithms that are used in a particular sequence. The algorithms have been implemented in a MATLAB environment. In the first step, the noise that might be originating from the imaging sensor and/or the optical path in a raw image is suppressed by applying a Wiener filtering technique [25]. In the second step, the denoised image is further processed by identifying and localizing several co-variant regions called Maximally Stable Extremal Regions (MSERs) in the image [26]. In the third step, a filter is applied to select a representative MSER from the detected co-located MSERs. In the fourth step, a binary image is generated by setting the selected regions in the image to one and keeping the other regions to zero. In the final step, some geometrical and spatter-related features are extracted from the binarized image. Each step is briefly explained in the forthcoming subsections.

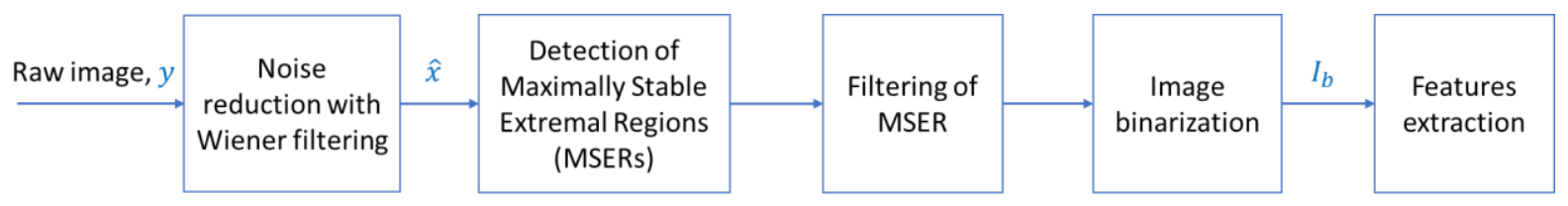

Figure 3. Flowchart of the image processing algorithm for spatter detection.

\subsubsection{Wiener filtering for image denoising}

Suppose that a measured image is corrupted by independent zero-mean white Gaussian noise. The problem can be formulated as follows:

$$
y(n, m)=h(n, m) * x(n, m)+v(n, m)
$$

Where $y(n, m)$ denotes the measured image with $n$ and $m$ being the pixel indices, $h(n, m)$ denoting the linear time-invariant impulse response function of the optical path, $x(n, m)$ is the (unknown) true image, the operator * denoting convolution and $v(n, m)$ denoting the measurement noise. 
The objective is to remove noise or denoise $y(n, m)$ and to obtain an estimate $\hat{x}(n, m)$ of $x(n, m)$, which minimizes the mean squared error (MSE),

$$
\operatorname{MSE}(\widehat{\boldsymbol{x}})=\frac{1}{N} \sum_{n, m=1}^{N}(\hat{x}(n, m)-x(n, m))^{2}
$$

where $N$ denotes the number of pixel elements in $x(n, m)$.

When $x(n, m)$ and $v(n, m)$ are stationary Gaussian processes, the Wiener filter is the optimal filter [25]. Specifically, when $x(n, m)$ is also a white Gaussian process, the Wiener filter can be simply formulated as follows:

$$
\hat{x}(n, m)=\mu_{x}(n, m)+\frac{\sigma_{x}^{2}(n, m)}{\sigma_{x}^{2}(n, m)+\sigma_{n}^{2}(n, m)}\left[y(n, m)-\mu_{x}(n, m)\right]
$$

Where $\sigma^{2}$ and $\mu$ are the image variances and means, respectively. To use Eq. (4), the image mean $\mu_{x}(n, m)$, the image variance $\sigma_{x}^{2}(n, m)$ and the noise variance $\sigma_{n}^{2}(n, m)$ need to be determined. If the noise variance is unknown, it is taken equal to the average of all the local estimated variances. The image mean and variance are calculated locally around each pixel over a uniform moving average window of size $(2 r+1) \times(2 r+1)$ :

$$
\begin{gathered}
\hat{\mu}_{x}(n, m)=\frac{1}{(2 r+1)^{2}}\left[\sum_{p=n-r}^{n+r} \sum_{q=m-r}^{m+r} y(p, q)\right] \\
\hat{\sigma}_{x}^{2}(n, m)=\frac{1}{(2 r+1)^{2}}\left[\sum_{p=n-r} \sum_{q=m-r}^{m+r}\left(y(p, q)-\hat{\mu}_{x}(n, m)\right)^{2}-\sigma_{n}^{2}(n, m)\right]
\end{gathered}
$$

With the parameter $r$ denoting an integer value. The choice of the parameter $r$ for determining the window size for averaging depends on the image size. Moreover, the choice is applicationspecific and should be determined experimentally. However, according to [26], the value of the parameter $r$ should be selected around 2 or 3 .

\subsubsection{Detection of Maximally Stable Extremal Regions (MSERs)}

Maximally Stable Extremal Regions (MSERs) are connected areas characterised by almost uniform intensity, surrounded by contrasting backgrounds. MSERs are constructed through a process of trying multiple thresholds. The selected regions are those that maintain unchanged shapes over a large set of thresholds [27]. The concept of MSER can be explained as follows. Assume that there exist all possible thresholds of a grey-level image $I=\hat{x}$. Define pixels below a threshold as 'white' and those above or equal to the threshold as 'black'. If thresholded images $I_{t}$ are shown one after the other, with time frame $t$ being also the threshold $t$, a black image will first appear at $t=0$. Subsequently, white spots corresponding to local intensity minima will appear and grow. At some point, regions corresponding to local minima will merge, enclosing black regions of high value pixels. For increasing threshold, these black regions will shrink. Finally, 
the last image will be completely white. The set of all connected components of all frames of the movie is the set of all maximal regions (MSER 1, MSER $2, \ldots, \operatorname{MSER} k, \ldots, \operatorname{MSER} N$ ), as seen in Figure 4 (b). The reader is referred to [27] for a more thorough description of the method.

\subsubsection{Filtering}

In case where some MSERs are co-located, for example, MSER $k$ with MSER $k+1$, and MSER $N-1$ with MSER $N$ as illustrated in Figure 4 (b), filtering needs to be applied to select the most representative MSER. The first step is to check whether co-located MSERs exist. To this end, an MSER with the largest area is selected as a reference MSER. Then, the distance between the centroid of a benchmarked MSER and that of the reference MSER is calculated. If the centroid distance is smaller than a certain threshold, the benchmarked MSER is thus colocated with the reference MSER. The second-largest area of the co-located MSERs is selected as a representative MSER. Based on the illustrative example shown in Figure 4 (b), the representative MSER for the two co-located MSER $k$ and MSER $k+1$ is MSER $k$, while the representative one for the two co-located MSER $N-1$ and MSER $N$ is MSER $N-1$.

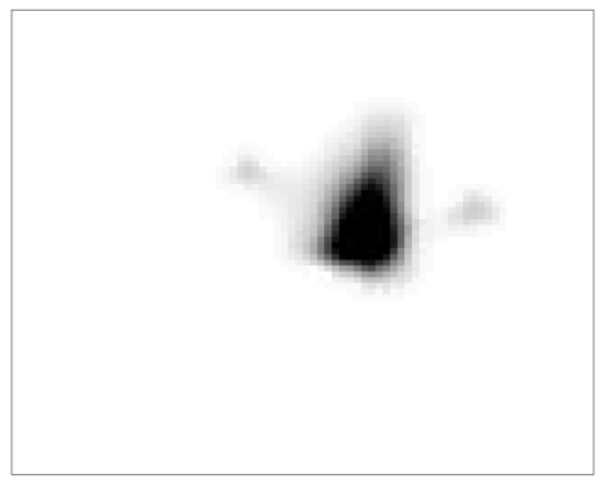

(a)

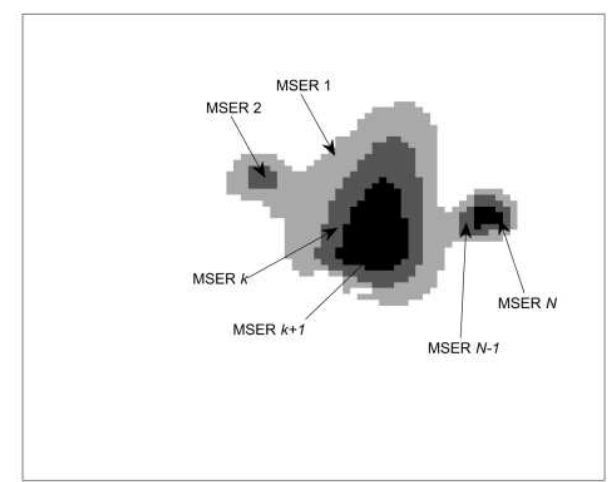

(b)
273

Figure 4. An illustrative example of identification of MSERs from an image: (a) raw/preprocessed image, (b) MSERs identified from the image.

\subsubsection{Image binarization}

Once co-located and non-co-located MSERs are selected, the binary image $I_{b}$ is simply generated by setting the pixel values on the selected MSERs to one (black), and by setting the pixel values of the background to zero (white).

\subsubsection{Features calculation}

Figure 5 illustrates several features that are extracted from the binary image $I_{b}$. The largest object detected in the binary image $A_{0}$ is taken as the melt pool, while the smaller objects $\left(A_{1}, \ldots, A_{j}, \ldots, A_{N}\right)$ are taken as spatters. The first defined features are the length, width and area. They are extracted using Hu's Moment Invariants [28] as implemented in the MATLAB 
environment. The area is calculated by taking the $0^{\text {th }}$ moment, which is the equivalent to the total number of pixels of the original object. To determine the length and width, an ellipse that has the same normalized second central moments as the segmented object is construed for each object, as can be seen in Figure 5. The size of the principal axes of the equivalent ellipses then represent the length and width (major axis (a) and minor axis (b) respectively). The second feature is the main melt pool eccentricity, $\varepsilon=\sqrt{1-\frac{b^{2}}{a^{2}}}, b<a$. The third feature is the perimeter of the main melt pool $\rho$. The fourth feature is the number of spatter, $N$. The fifth feature is the spatter ratio $r$ being defined as the division between the total area of spatters and the main melt pool area, $r=\frac{\sum_{j=1}^{N} A_{j}}{A_{0}}$. The first 3 features are expressed as scalars in the unit of pixel. The remaining 2 , number of spatter and spatter ratio, are dimensionless.

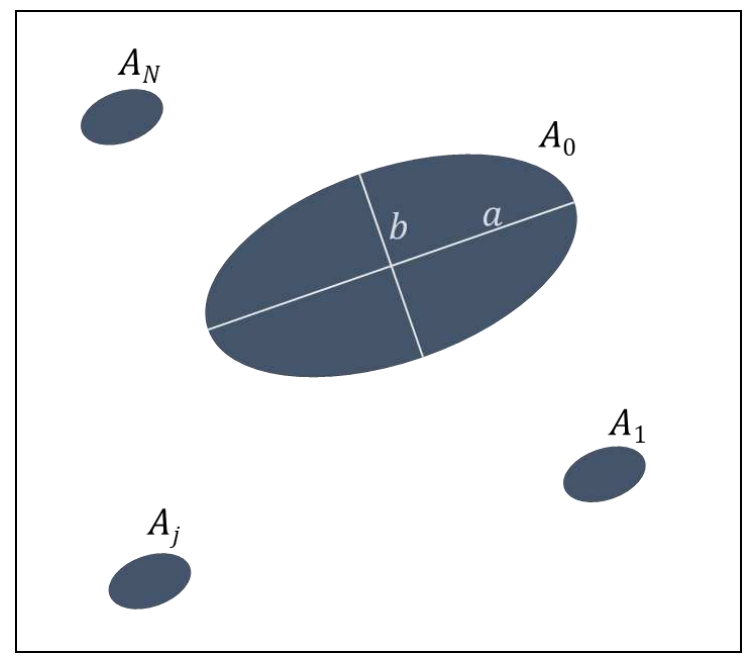

Figure 5. Illustration of features calculated from a binary image $I_{b}$.

\section{$2.4 \quad$ X-ray computed tomography}

X-ray computed tomography (X-CT) measurements are performed to scan the object with a XT H 225 ST machine from Nikon Metrology having a maximum tube potential of $225 \mathrm{kV}$. The instrumental parameters are set at a tube voltage of $180 \mathrm{kV}$, a power of $12.1 \mathrm{~W}$, an exposure time of $2000 \mathrm{~ms}$ and 3600 radiographs. The chosen target material for the X-ray generation is tungsten. The magnification is fixed at a factor of 20, prompting a measurement resolution (voxel size) of $10 \mu \mathrm{m}$. A copper filter of $1 \mathrm{~mm}$ thickness is utilized in front of the X-ray tube window to reduce the beam hardening effect. The volume reconstruction is performed using the Feldkamp-Davis-Kress algorithm. The alignment of the reconstructed volume is performed by the 3-2-1 registration technique with VGStudio MAX 3.2 software.

\subsection{Numerical modelling of the melt pool}

A Finite Volume Method based Computational Fluid Dynamics (CFD) model introduced by Bayat et al., 2019 [12] is used to simulate the heat and fluid flow conditions for both optimum and keyhole LPBF conditions. The model solves the conservation of mass, linear momentum and energy equations. It uses the Fresnel absorption function to model the laser behaviour [29], 
which is dependent on the geometry of the depression zone. The model also takes into account several physical based phenomena such as evaporation, ablation pressure, melting, capillarity, Marangoni effect, solidification and the laser-material interaction [30]. Besides, it encompasses the ambient heat losses through the exposed free surfaces via convection, radiation and evaporation.

\section{Results and discussion}

The first part of this section shows the geometrical features of a melt pool comprising of the major axis-length, the minor axis-width and the melt pool area, as measured from the coaxial camera melt pool images of the single track experiment. The second part discusses the features comprising the number of spatters, the spatter area and the spatter ratio of the single track experiment. The third part presents numerical modelling results of the melt pool for optimum and keyhole LPBF conditions. In the final part of this section, melt pool geometry features are evaluated from optical micrographs.

\subsection{Geometrical melt pool features for the single track experiment}

This section shows the results from the photodiode and the high-speed camera for single tracks. The photodiode indicates the average melt pool intensity (single value output) and the highspeed coaxial camera records the melt pool geometry (2D array of pixels) from which melt pool characteristics like length and width are extracted. Table 2 shows the melt pool intensity for line scans with different processing parameters. The average intensity of six line-scans is presented with its associated standard deviation. The first up to 15 data points in the beginning and up to 10 data points at the end of the line scan are neglected due to the non-uniformity of the applied energy density. This is a result of the acceleration and deceleration of the galvanometer mirrors. The melt pool emission energy is calculated by averaging the photodiode signal and subsequently integrating over time, for a certain line length e.g. $1000 \mathrm{~mm}$. This integrated value logically increases with decreasing scan speed (longer duration) if the average emitted intensity remains the same.

However, it is clear from Table 2 that the average melt pool emission intensity does not scale linearly with the volumetric energy density (VED). Indeed, it is found that the recorded average signal from the photodiode for condition 2 is $14.7 \%$ higher than for optimum condition 1 . While for condition 3 the recorded average signal is $13.2 \%$ lower than what was found for condition 1 , even though both are observed to have the same VED. Alternatively, examining the average integrated melt pool intensity, condition 3 now exhibits the highest value. For condition 3 the integrated average value is $73.5 \%$ higher compared to condition 1 . In contrast, condition 2 exhibits the same $14.7 \%$ increase in integrated average value compared to optimum condition 1. These results could indicate that the occurrence of keyhole mode melting (condition 3) is tied with the integrated average of the melt pool radiation, while simultaneously it is less dependent on the average measured radiation. Figure 6 shows examples of the recorded melt pool intensity for a single track with optimum, high and low VED conditions. 

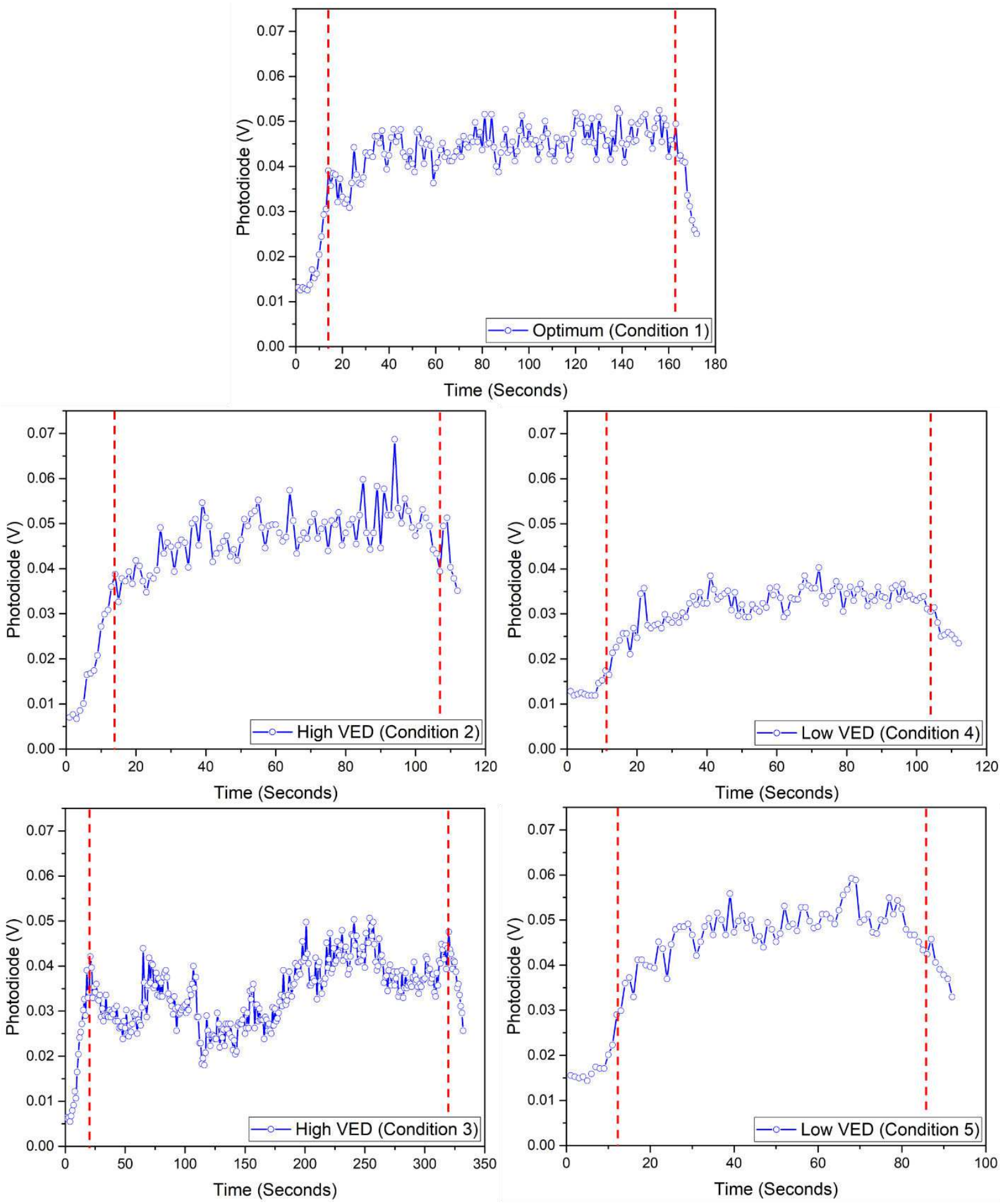

Figure 6. Melt pool intensity (photodiode signal (V)) of single track with optimum, high and low VED conditions. Data between two dashed red lines are taken into account. 
Table 2. Melt pool (MP) Intensity from Photodiode for LPBF of Ti-6Al-4V.

\begin{tabular}{|c|c|c|c|c|c|}
\hline $\begin{array}{c}\text { Condition and } \\
\text { VED }\end{array}$ & ID & $\begin{array}{c}\text { Laser } \\
\text { Power / W }\end{array}$ & $\begin{array}{c}\text { Scan Speed / } \\
\mathbf{m m} \cdot \mathbf{s}^{-1}\end{array}$ & $\begin{array}{c}\text { Average MP } \\
\text { Intensity / mV }\end{array}$ & $\begin{array}{c}\text { Integrated } \\
\text { intensity over } \\
\text { time for a } \\
\text { length of 1000 } \\
\mathbf{m m} / \mathbf{~ V} \cdot \mathbf{s}\end{array}$ \\
\hline Optimum VED & 1 & 170 & 1000 & $40.1 \pm 1.5$ & 0.0401 \\
$\left(151 \mathrm{~J} \cdot \mathrm{mm}^{-3}\right)$ & 2 & 340 & 1000 & $46.0 \pm 1.7$ & 0.0460 \\
\hline High VED & 3 & 170 & 500 & $34.9 \pm 0.7$ & 0.0696 \\
\cline { 2 - 6 }$\left(302 \mathrm{~J} \cdot \mathrm{mm}^{-3}\right)$ & 4 & 85 & 1000 & $29.7 \pm 1.9$ & 0.0297 \\
\hline Low VED & 5 & 170 & 2000 & $43.4 \pm 1.7$ & 0.0216 \\
\cline { 2 - 6 }$\left(75.6 \mathrm{~J} \cdot \mathrm{mm}^{-3}\right)$ & 5 & & & & \\
\hline
\end{tabular}

354

355

356

357

358

359

360

361

362

363

364

365

366

367

368

369

370

371

372

373

374

375

376

377

In order to extract the melt pool geometry from the grayscale camera images, the camera images are pre-processed using the processing steps detailed in Section 2.3 to generate binary images. Some geometrical features including the melt pool length, width and area are calculated in units of pixels. For the single track experiments, the pixel size is equal to roughly $14 \mu \mathrm{m}$ after camera calibration. Figure 7 shows the different melt pool features for an optimum condition (condition $1, P=170 \mathrm{~W}, v=1000 \mathrm{~mm} \cdot \mathrm{s}^{-1}$ ) and Figure 8 shows the different melt pool features for a high VED (condition 3, $P=170 \mathrm{~W}, v=500 \mathrm{~mm} . \mathrm{s}^{-1}$ ). The average melt pool geometry results of all six line scans with optimum and keyhole conditions are presented in Table 3. As can be seen from the recorded and average melt pool dimensions, no significant difference in the melt pool area, width and length for the optimum (condition 1) and the keyhole mode (condition 3) is observed. While the average melt pool length is shorter and the width is larger for condition 3 as compared to condition 1, the variations are larger than the difference in dimensions, making the difference statistically insignificant.

Figure 7 shows a very stable melt pool during the optimum process parameters (condition 1). In Figure 8 however, an unstable melt pool, as well as melt pool anomalies, are observed during the line scan for condition 3. These anomalies are due to spatter ejecting from the melt pool during processing. Indeed, a peak in the melt pool area and perimeter does not necessarily mean that the melt pool grows to be very large. Instead due to the 2D representation of a 3D space, both the spatter and the melt pool appear to be connected resulting in a large apparent area. In reality, the spatter is situated in a plane above that of the melt pool. As soon as the spatter detaches from the melt pool in the camera image, the melt pool area immediately drops back to the expected level (for an illustration see last three pictures in Figure 13). In the next section, results from the image processing algorithm for spatter detection are explained in detail after discussing possible spatter formation mechanisms. 

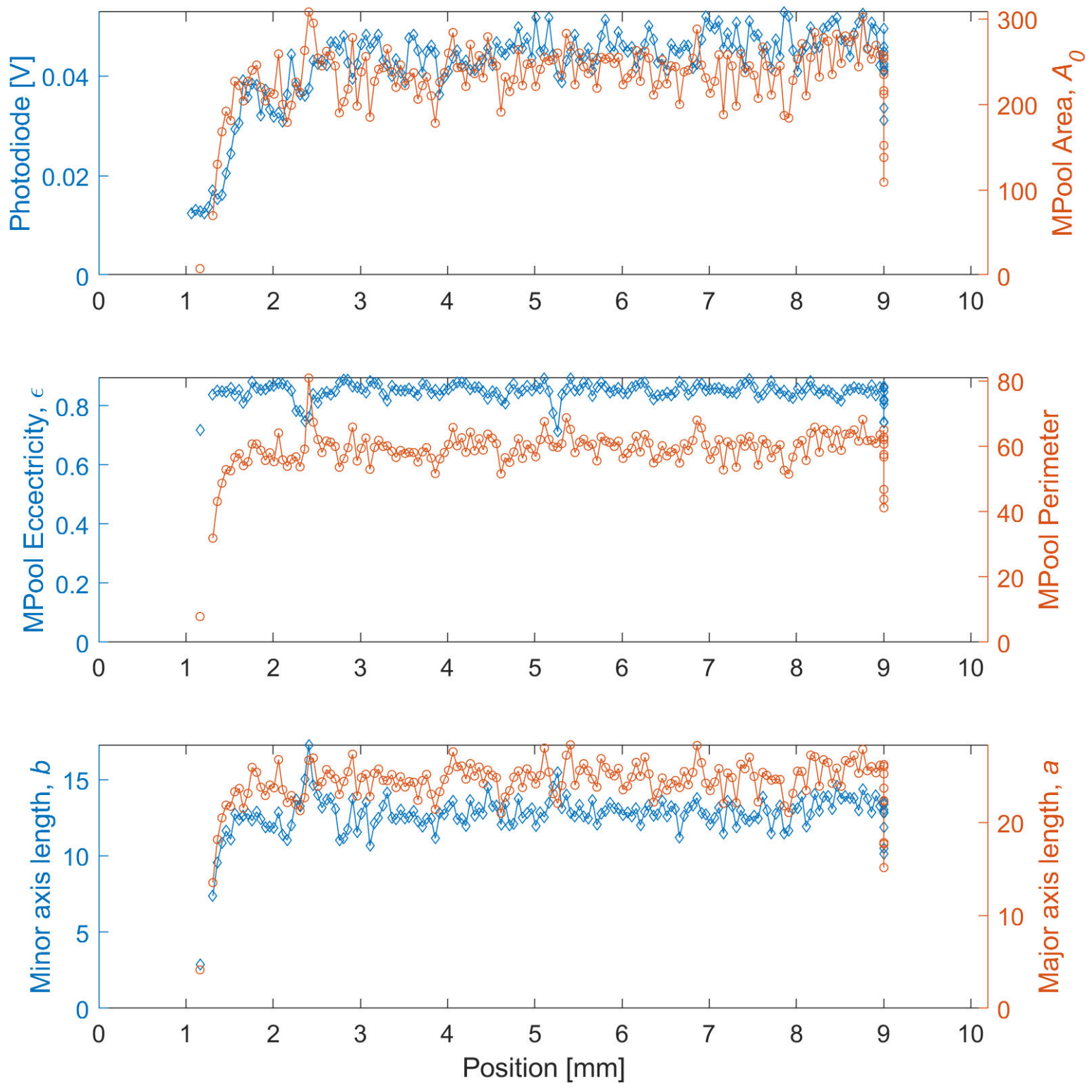

378

379 Figure 7. Melt pool features for the optimum condition (condition 1, $P=170 \mathrm{~W}, v=1000 \mathrm{~mm} . \mathrm{s}$ 380

$1)$. 

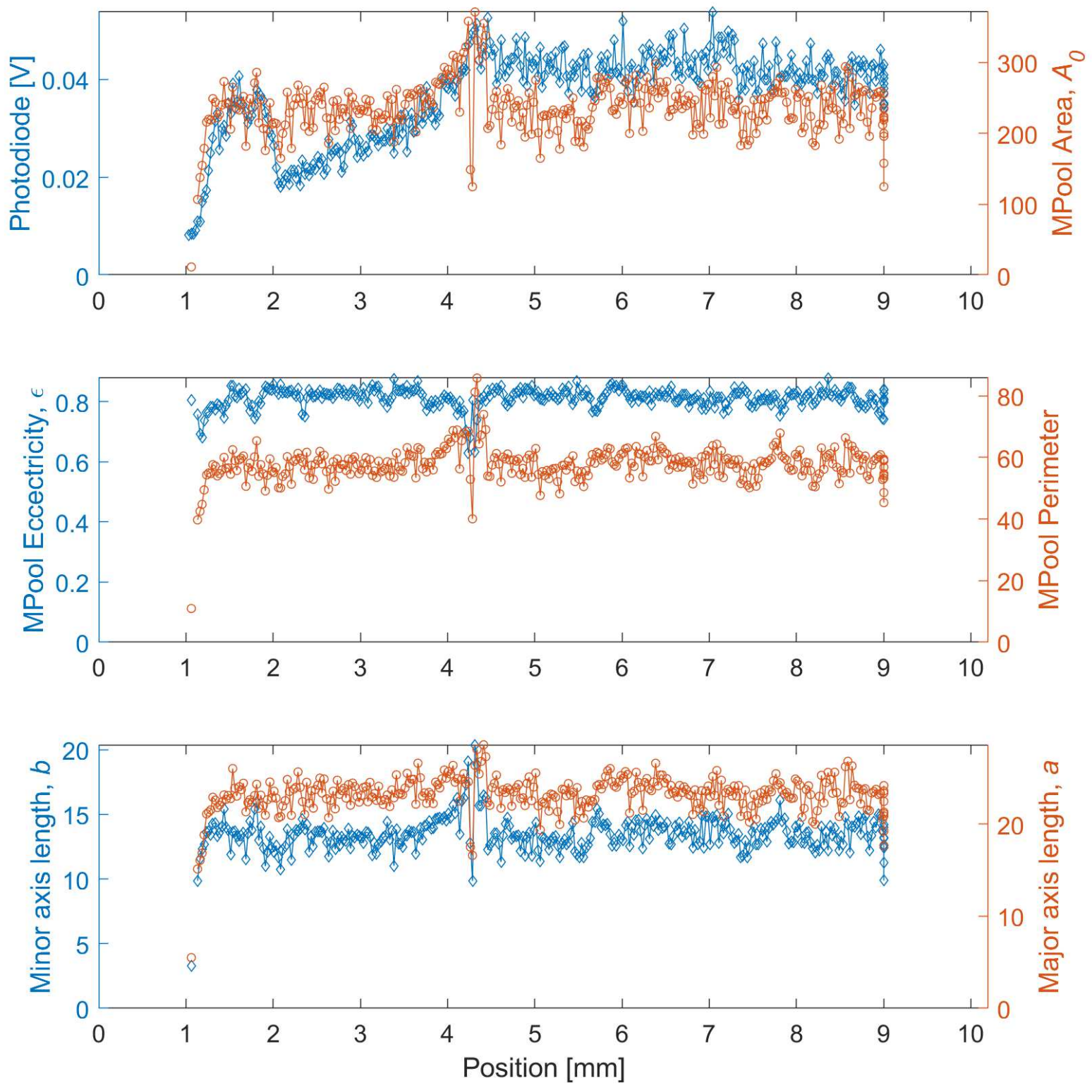

381

382 Figure 8. Melt pool features for a high VED (condition $3, P=170 \mathrm{~W}, v=500 \mathrm{~mm} \cdot \mathrm{s}^{-1}$ ).

383

384

385 
Table 3. The statistics of the melt pool geometrical features of optimum and keyhole conditions for LPBF of Ti-6Al-4V calculated from the position of $2 \mathrm{~mm}$ to $8 \mathrm{~mm}$ as shown in Figure 7 and 8.

\begin{tabular}{|c|c|c|c|c|c|c|}
\hline Condition & ID & $\begin{array}{c}\text { Melt pool } \\
\text { width / pixel }\end{array}$ & $\begin{array}{c}\text { Melt pool } \\
\text { length / pixel }\end{array}$ & $\begin{array}{c}\text { Area / } \\
\text { pixel }^{2}\end{array}$ & $\begin{array}{c}\text { Perimeter / } \\
\text { pixel }\end{array}$ & $\begin{array}{c}\text { Eccentricity } \\
/\end{array}$ \\
\hline $\begin{array}{c}\text { Optimum } \\
\text { VED }\end{array}$ & 1 & $12.8 \pm 0.9$ & $24.6 \pm 1.6$ & $237 \pm 25$ & $59 \pm 4$ & $0.85 \pm 0.03$ \\
\hline $\begin{array}{c}\text { High } \\
\text { VED }\end{array}$ & 3 & $13.6 \pm 1.3$ & $23.5 \pm 1.6$ & $241 \pm 34$ & $58 \pm 5$ & $0.81 \pm 0.03$ \\
\hline
\end{tabular}

\subsection{Spatter features for the single track experiment}

The formation of spatter is a well-studied subject. Nevertheless, the correlation between the keyhole pore generation and the spatter formation observed in coaxial images remains unclear. A recent study by Young et al., 2020 [31], utilizing high speed x-ray imaging, distinguishes 5 types of spatters. Of these 5 types, only 2 are directly related to melt pool instabilities. Furthermore, they show that some types of spatters are always present irrespective of the processing conditions or melting mode. Wang et al., 2017 [32] distinguishes three types of spattering, mainly caused by melt pool instabilities due to Marangoni effect, recoil pressure and heat effect in molten pool. In general, two mechanisms of spatter formation are observed during keyhole mode melting. The first is related to the melt pool collapse as discussed before. The sudden breakdown of the keyhole is accompanied with a large change in the melt velocity and part of this melt can be pinched off during the collapse. This liquid metal stream elongates and breaks up into smaller droplets to minimize surface energy [9]. The second mechanism relates to the metallic jet stream coming from the keyhole because of the recoil pressure. The metallic vapour (typically ejected towards the rear) generates shear forces on the rear keyhole wall. These forces can reach such high levels that they can overcome the surface tension. As a result, liquid detachment can occur in the rear of the melt pool, generating spatter. Gunenthiram et al., 2018 [20] investigated and quantified the formation of spatters during an LPBF process and addressed the possible influence of keyhole formation on such instabilities. A correlation was proposed between volumes of spatters and volumetric energy density (VED). Repossini et al., 2017 [21] also showed that the over-melting condition produce a larger number of spatters than the nominal melting condition. In the analysis done in this work, we will investigate spatter formation for conditions 2 and 3 that have identical high VED.

By employing a novel spatter detection workflow on high-speed coaxial images, a correlation can be made between the post-process X-CT observed keyhole porosity and the detected spatter in the images. Figure 9 and 10 show representative examples of the raw and processed coaxial melt pool images both in the absence and presence of spatters. The presence of spatters is not so obvious in the raw image, as shown in Figure 10 (a). Nevertheless, the spatters in the latter figure are effectively enhanced by the proposed algorithm, see Figure 10 (b). 


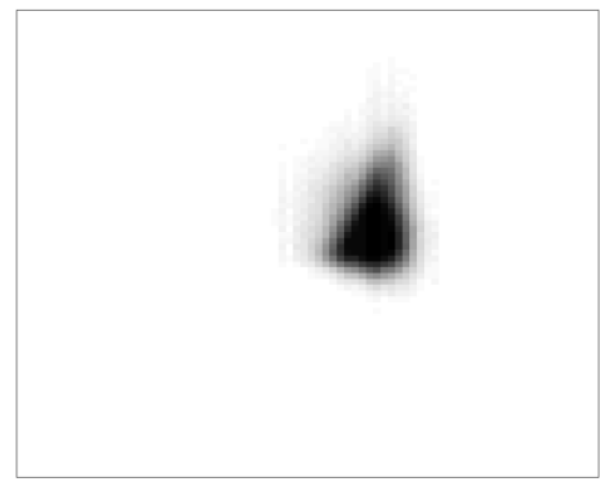

(a)

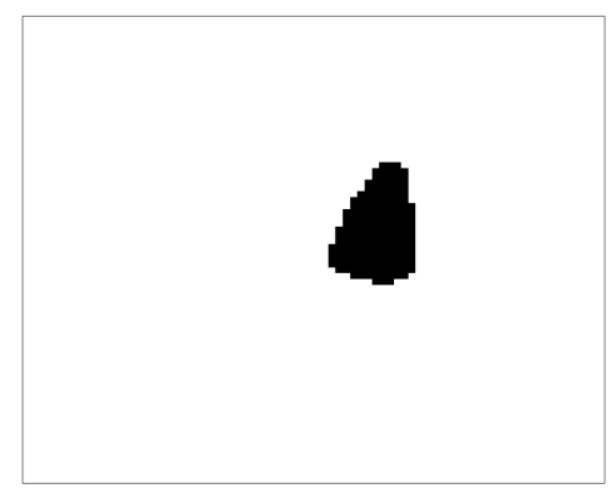

(b)

418 Figure 9. An example of a processed image in the absence of spatters: (a) a raw image acquired processing algorithm to the raw image.

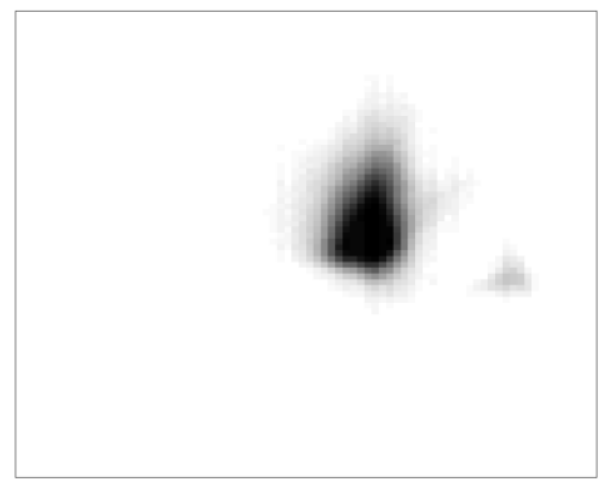

(a)

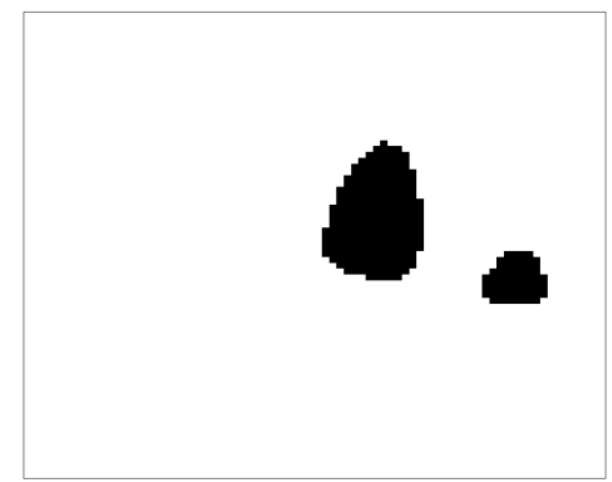

(b)

421 Figure 10. An example of a processed image in the presence of spatters: (a) raw image acquired processing algorithm to the raw image. 

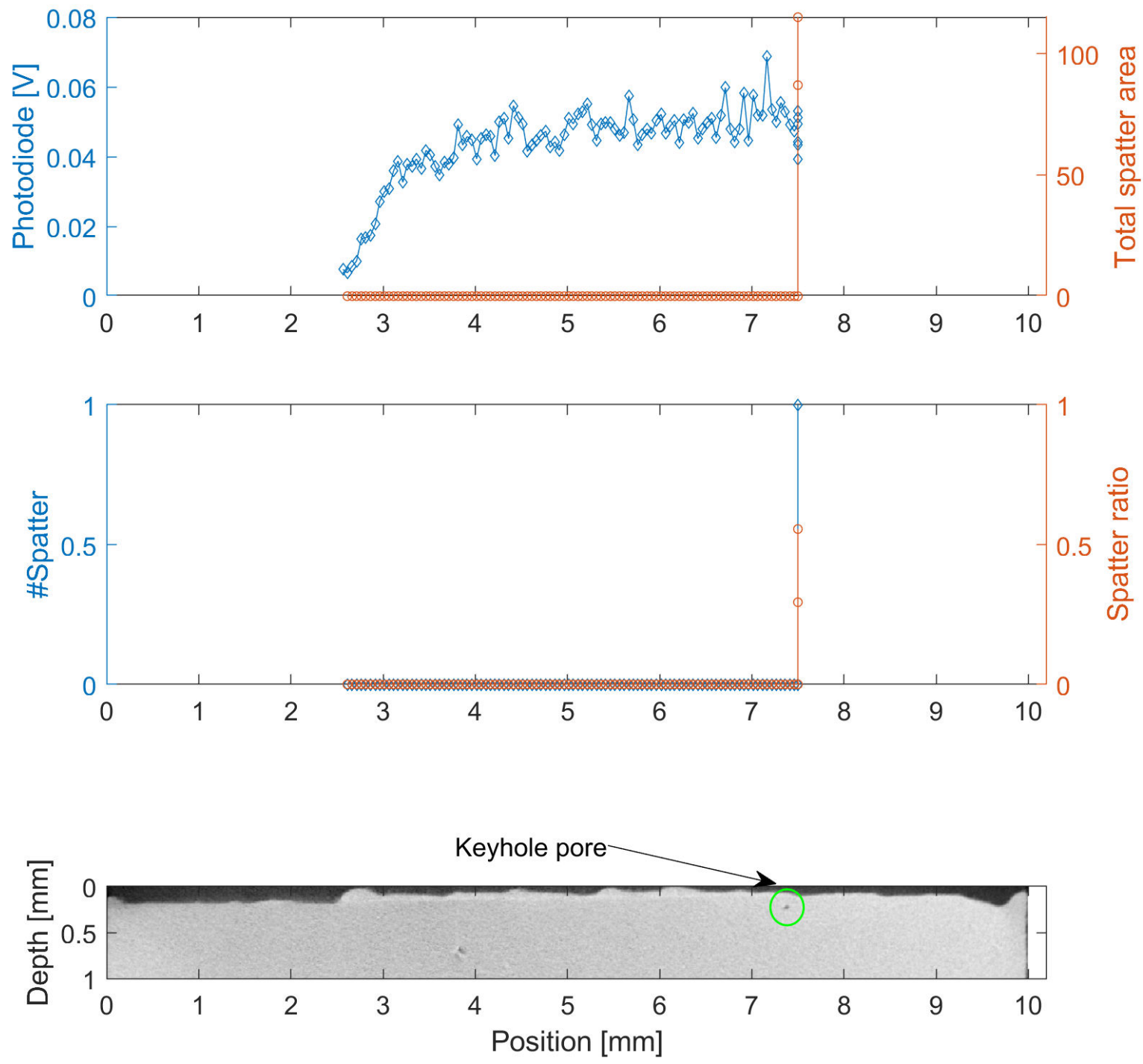

Figure 11. Comparison of spatter data calculated from melt pool images and porosity information obtained from X-CT for high VED (condition 2, $P=340 \mathrm{~W}, v=1000 \mathrm{~mm} \cdot \mathrm{s}^{-1}$ ). 

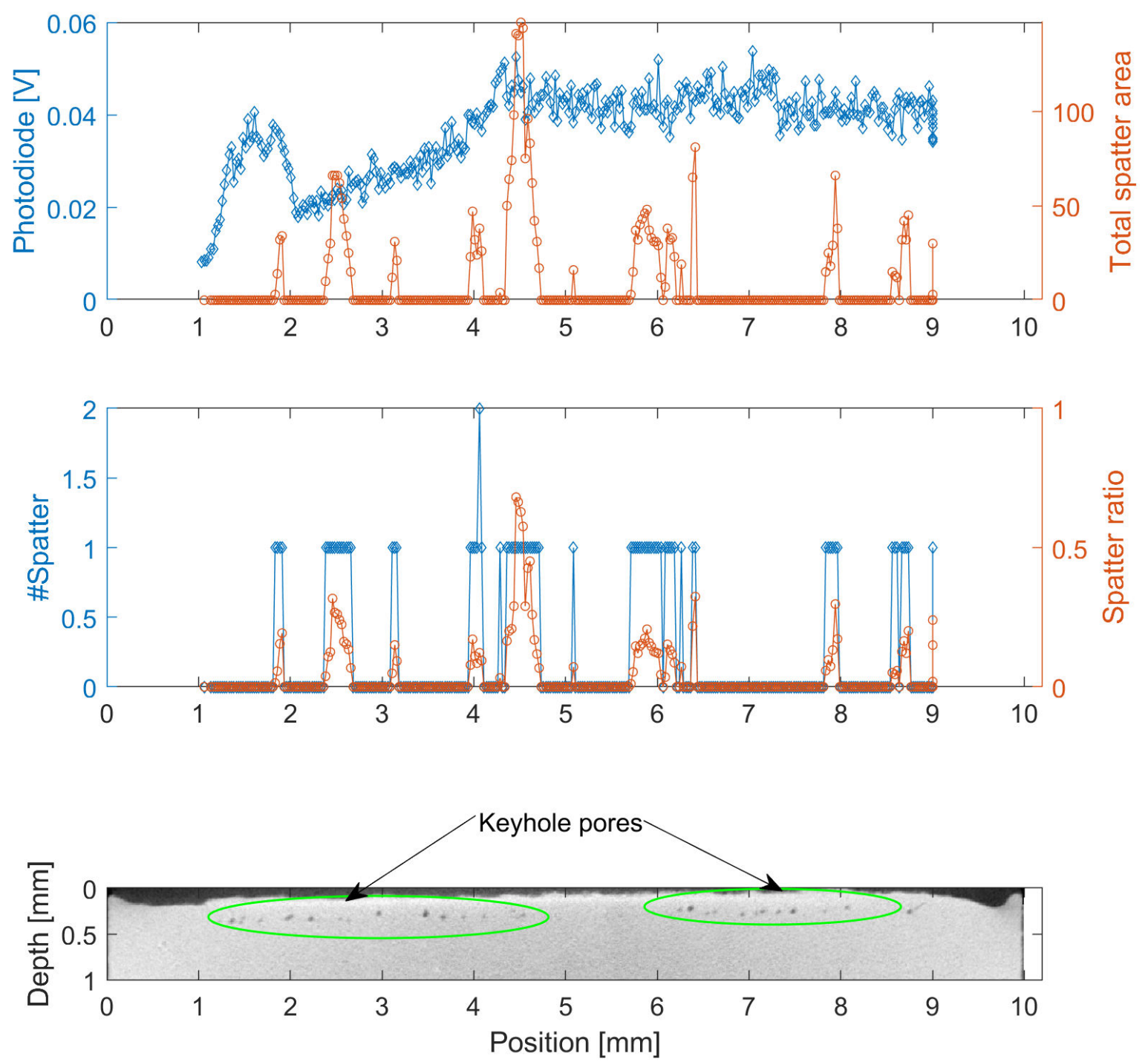

Figure 12. Comparison of spatter data calculated from melt pool images and porosity information obtained from X-CT for high VED (condition $3, P=170 \mathrm{~W}, v=500 \mathrm{~mm} \cdot \mathrm{s}^{-1}$ ).

Figure 11 and 12 show the number of spatters, extracted from the melt pool images for the high VED conditions 2 and 3. The transversal X-CT cross-sections are shown as well revealing the sub-surface keyhole porosity below the line scan. For condition 2, a keyhole pore is only formed at the end of the scan. This is in agreement with the observations of other authors [33], where the rapid collapse of the melt pool at the end of a track is associated with the formation of keyhole porosity. A keyhole at the end of the track can also be observed for condition 3. In both cases, the melt pool collapse can be associated with a spatter ejection. These observations confirm that the rapid melt pool closure on laser turn-off and galvanometer scanner deceleration is associated with keyhole porosity and spatter ejection, but furthermore, that these spatters are successfully detected by the algorithm applied to the camera images. 
Next to the keyhole at the end of the track, many more keyholes and significant spattering can be detected all along the track in the coaxial camera images for condition 3 (Figure 12). To further demonstrate the correlation between the formation of keyhole porosity and the presence of spatters, the subsequent binary melt pool images processed with the above described algorithm are shown in Figure 13 (condition 2) and 14 (condition 3). For condition 2, only 1 spatter event is seen in the last images. For condition 3, numerous spatters are observed over the entire length of the scan (for instance in the first image at $8.75 \mathrm{~mm}$ and last image at $9 \mathrm{~mm}$ in Figure 14), which might indicate a possible correlation with the many keyhole pores observed by $\mathrm{X}-\mathrm{CT}$ for this sample.

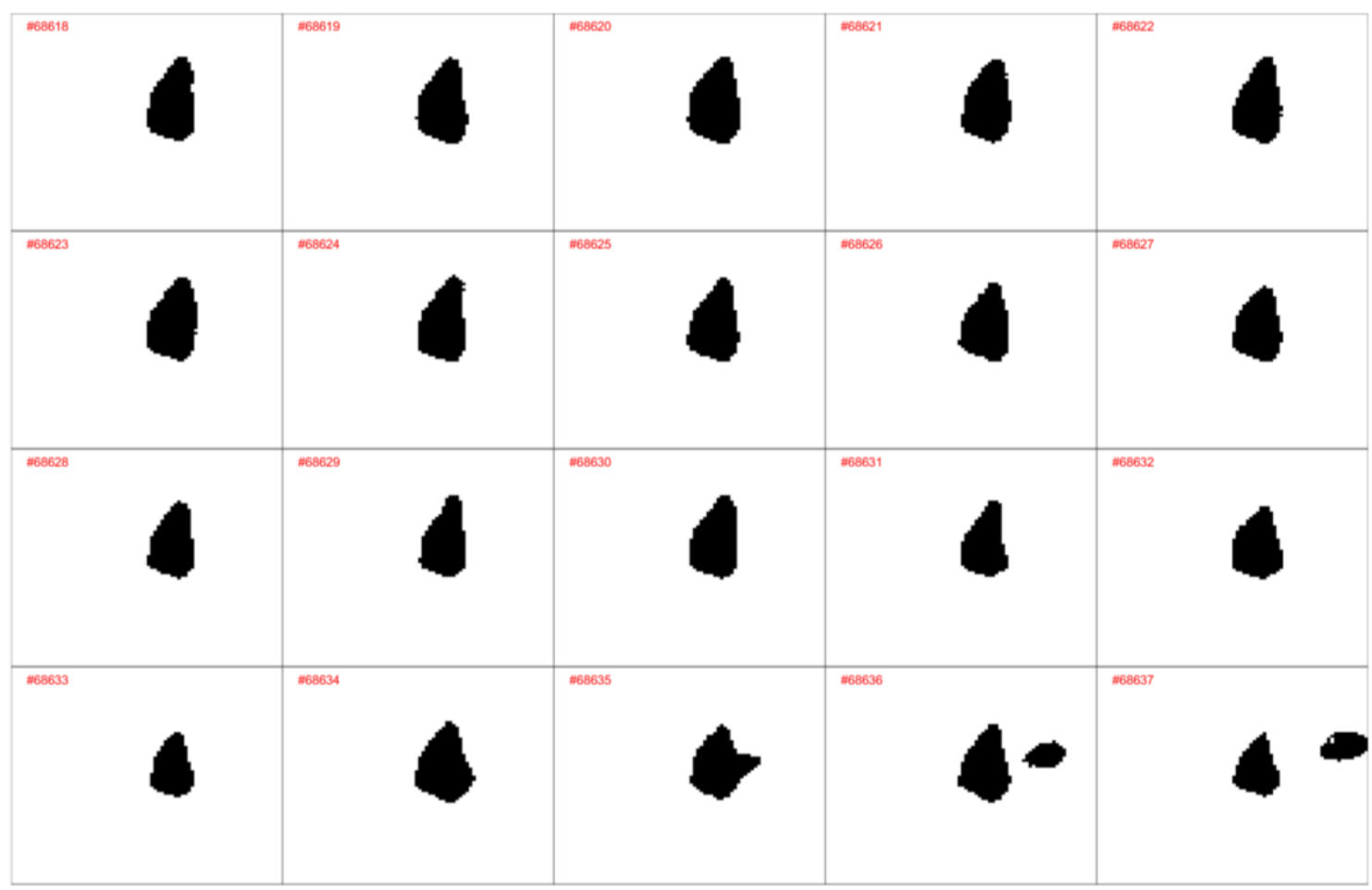

Figure 13. The evolution of the binarized melt pool images captured for the Position $\mathrm{Y}$ of 6.96 $\mathrm{mm}$ to $7.5 \mathrm{~mm}$ of the line scan with condition $2\left(P=340 \mathrm{~W}, v=1000 \mathrm{~mm} . \mathrm{s}^{-1}\right)$. 


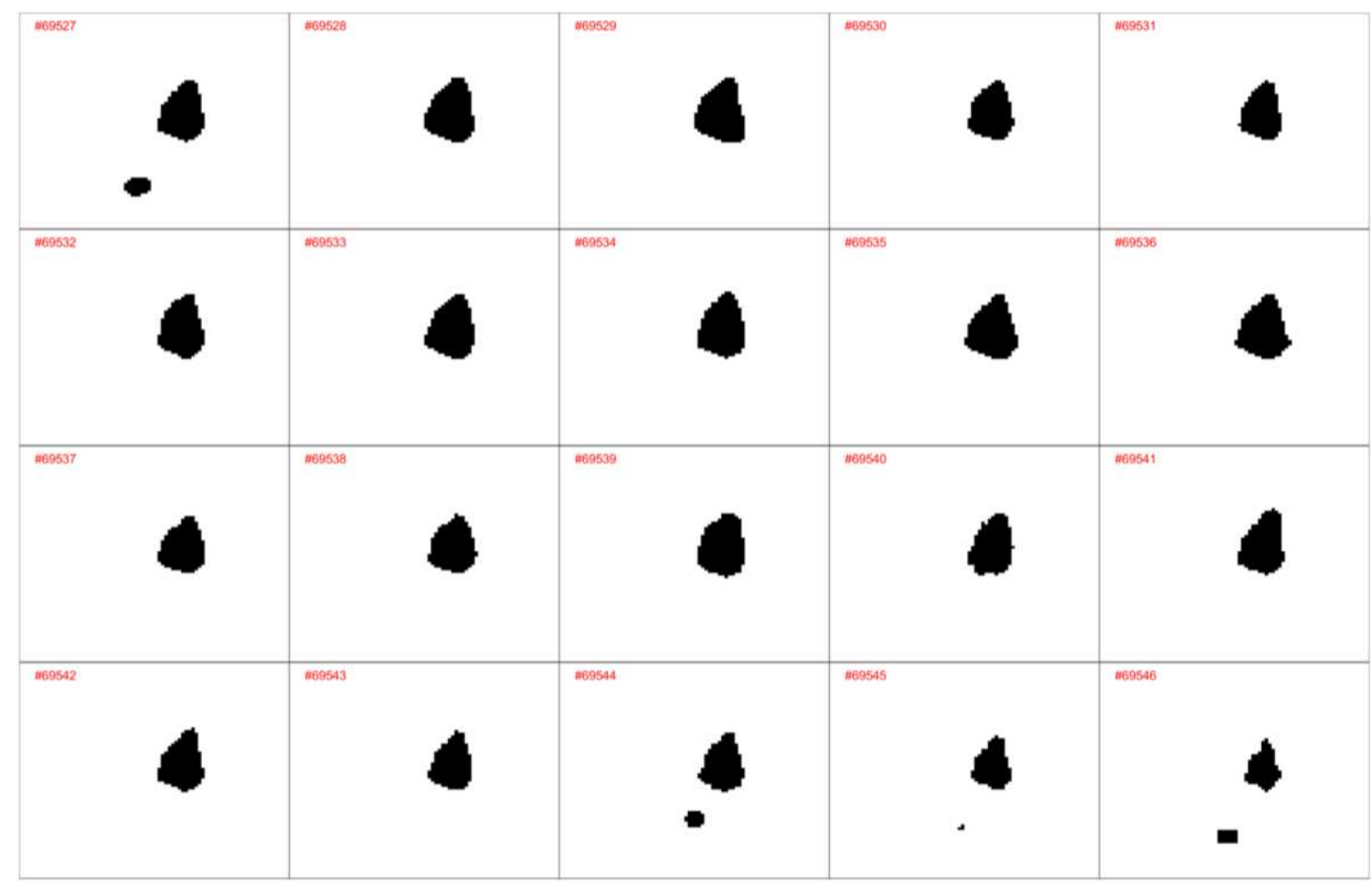

453 Figure 14. The evolution of the binarized melt pool images captured for the Position Y of 8.74 $\mathrm{mm}$ to $9 \mathrm{~mm}$ of the line scan with condition $3\left(P=170 \mathrm{~W}, v=500 \mathrm{~mm} . \mathrm{s}^{-1}\right)$.

455 To further investigate a possible correlation between the presence of spatters and the formation of keyhole pores, the melt pool images and the X-CT cross-section images of 12 line scans with high VED (condition 2 and 3) have been further processed and analysed. The analysis is performed from the start until the end of a single track with an analysis resolution of $100 \mu \mathrm{m}$. This means that if there are multiple spatters detected within a $100 \mu \mathrm{m}$ range, they are counted as a single spatter event. Figure 15 shows a correlation between the number of keyhole pores observed in the X-CT cross-section images and the number of the spatter events for all the line scans of condition 2 and 3. The horizontal axis of the plot represents the number of spatter events detected in the coaxial melt pool images, while the vertical axis represents the number of keyhole pores detected in the X-CT cross-section images. It is clear from Figure 15 that the number of spatter events correlates with the number of observed keyhole pores along the Ti-6Al-4V tracks studied in this work. 


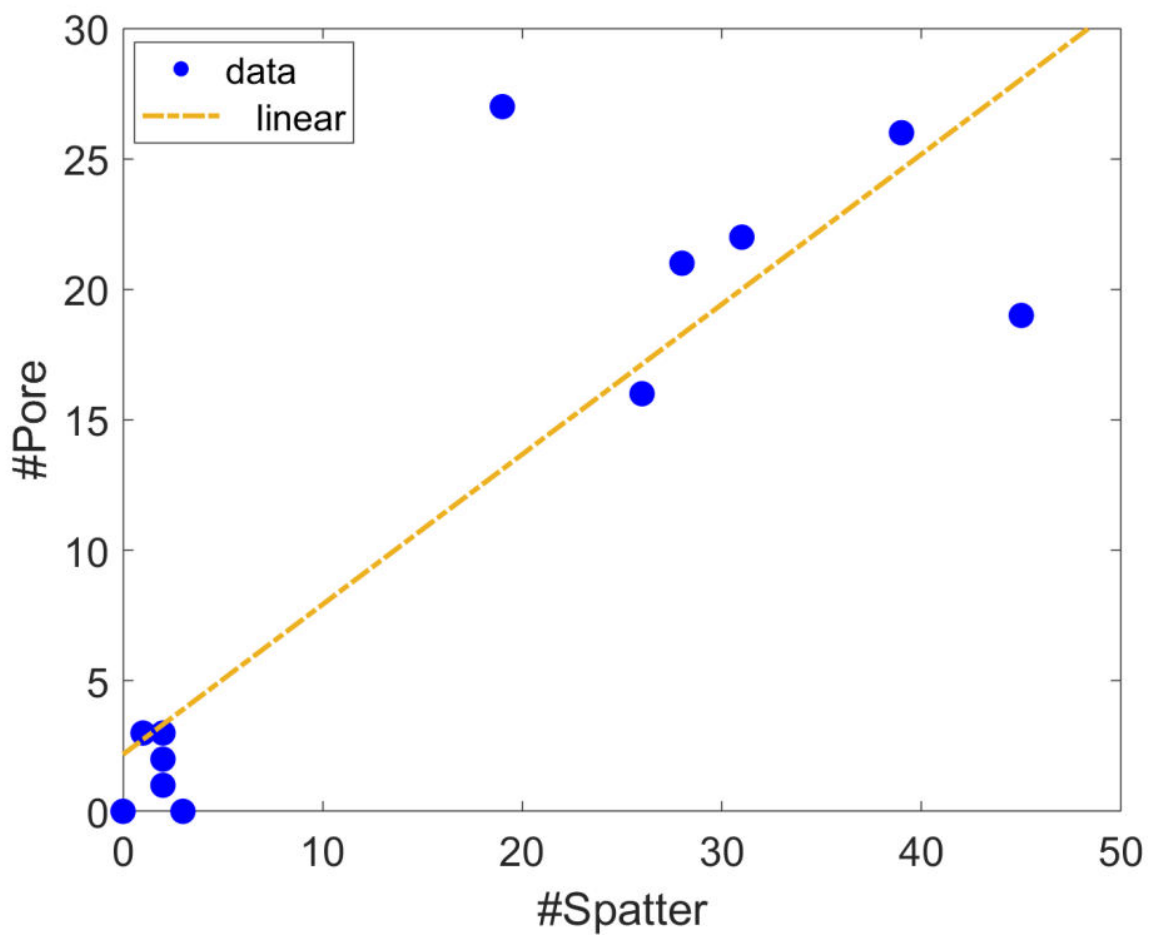

Figure 15. Correlation between the number of keyhole pores detected with the X-CT and the number of spatters detected with the camera images processed with the proposed algorithm obtained from the 12 line scans with high VED (condition 2 and 3). Pearson's linear correlation coefficient of such a relationship is 0.87 .

\subsection{Finite Volume Model of the melt pool}

With the melt pool monitoring system used in the previous sections, we can get information on spatter and melt pool width and length, but it is not possible to observe the melt pool depth. CFD simulations were therefore performed to get more insight in the dimensional evolution of all melt pool dimensions. The CFD simulation has been performed for two experimental conditions (condition 1: $P=170 \mathrm{~W}, v=1000 \mathrm{~mm} \cdot \mathrm{s}^{-1}$ and condition $3: P=170 \mathrm{~W}, v=500 \mathrm{~mm} \cdot \mathrm{s}^{-1}$ ). The computational domain is composed of $6,288,368$ cells with an average size of $3.3 \mu \mathrm{m}$. The cells are chosen with an aspect ratio very close to unity, which is preferred for surface-tracking algorithms. Top views of the simulated melt pool liquid fraction for both conditions are shown in Figure 16 (a) and (b). According to Figure 16 (a), the melt pool in the conduction mode is found to be in a stable condition due to its optimum VED level. In this case, the evaporation recoil pressure is not significant and thus the melt pool will not face any instability and will preserve its morphology as it moves along the track. 
(a)

$$
\text { Conduction mode }
$$

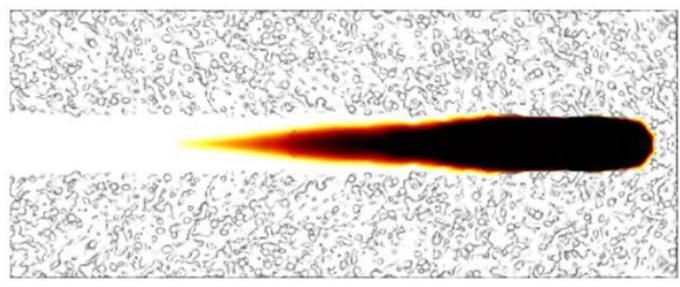

Liquid fraction (-)
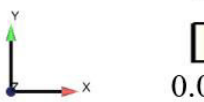

(b)

Keyhole mode

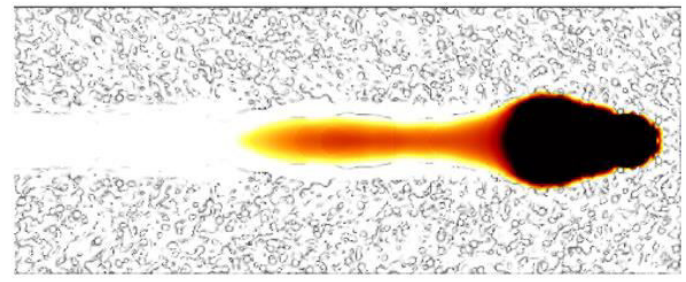

$100 \mu \mathrm{m} \longleftrightarrow$

Figure 16. The top surface of the melt pool after reaching the centre of the computational domain during the scanning of the single-tracks along with the liquid fraction contour for optimum condition (a) $1000 \mathrm{~mm} \cdot \mathrm{s}^{-1}$ and keyhole condition (b) $500 \mathrm{~mm} \cdot \mathrm{s}^{-1}$.

Based on Figure 16 (a) and (b), the melt pool in the conduction mode (optimum VED) is narrower and at the same time longer from the top view as compared to the melt pool in the keyhole mode (high VED). The melt pool in the keyhole condition is highly unstable and the depression zone fluctuates due to the competition of recoil pressure and surface tension. At the same time, the Marangoni effect is more pronounced in the keyhole condition, mainly due to higher temperature gradient levels. This effect will make the melt pool in the keyhole mode wider than in the conduction mode that has lower temperature gradients, thus less pronounced Marangoni effect. Based on Figure 16, the melt pool in keyhole mode is shorter and at the same time wider, compared to the conduction mode.

A more detailed modelled view of the melt pool's internal structure for both of the studied cases is given in Figure 17. According to Figure 17, it is noted that most of the melt pool in keyhole mode is situated substantially below the top surface of the bulk domain. Accordingly, the melt pool is observed to be shorter from the top view, as compared to the conduction mode. The melt pool, however, becomes a lot deeper in the keyhole mode as compared to the conduction mode. This depth cannot be observed in our in-process experiments. While the comparison between the top view results from the numerical model and the experiments show qualitative agreement, the melt pool geometry is however found to be smaller in the experiments. This can be due to the fact that in the numerical model, the effect of gas-phase and its possible effect on the laser beam attenuation is not considered. More importantly, the discrepancy can be attributed to the fact that in the experiments, the monitoring device cannot accurately locate the solidus line from the melt pool tail, mainly due to low radiation of this zone. In this respect, the melt pool length is underestimated in the experiments as compared to the numerical model.

It is found in previous work [12] that keyhole porosities can still travel, disappear or combine with other pores after formation, indicating that it proves very difficult to correlate melt pool signatures at a certain location with pores formed below. A correlation between the total number of spatter as seen from melt pool images and the total number of pores in a line of a certain length, as shown in Figure 15, is however still possible. 

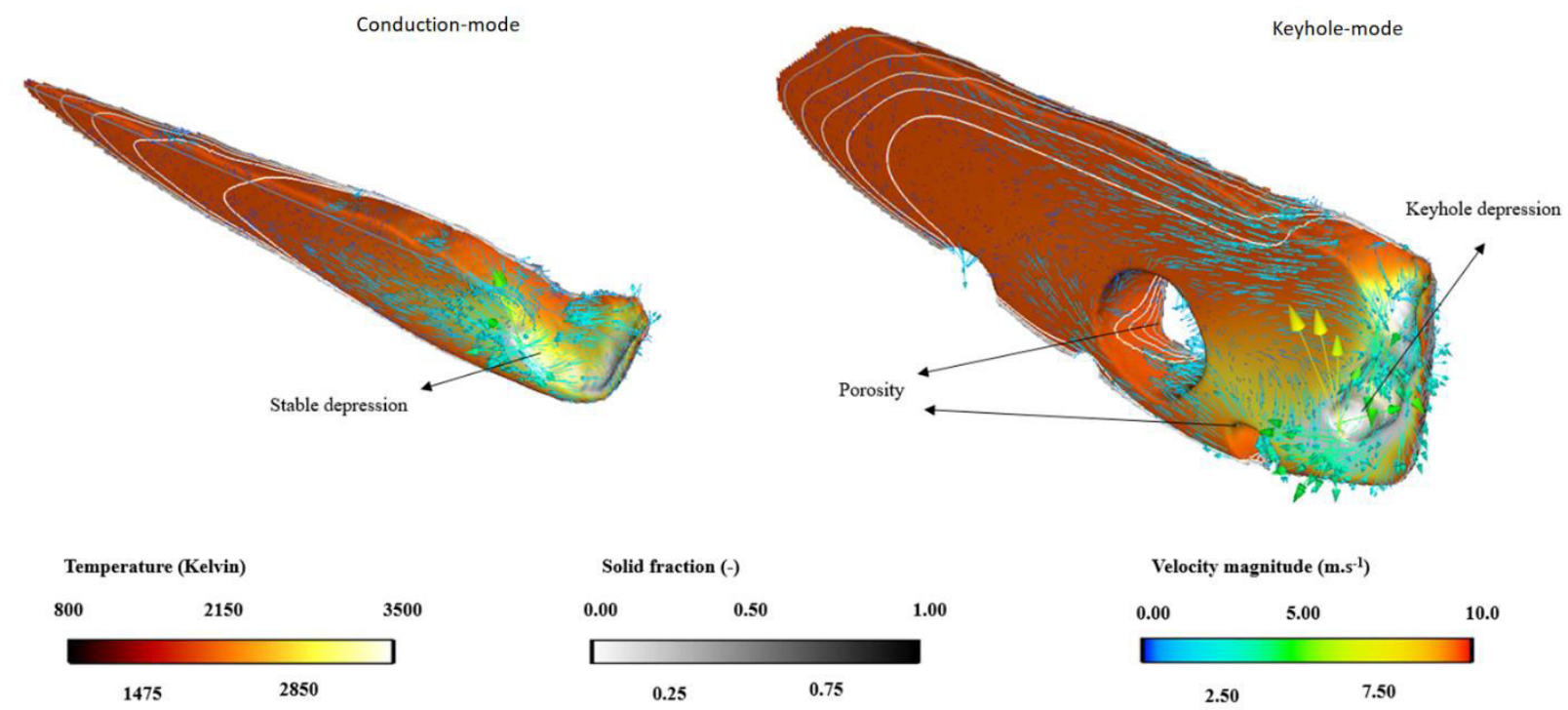

Figure 17. Melt pool anatomy after reaching the centre of the computational domain for optimum condition (left) with scan speed $1000 \mathrm{~mm} \cdot \mathrm{s}^{-1}$, and keyhole condition (right) with scan speed $500 \mathrm{~mm} \cdot \mathrm{s}^{-1}$.

\subsection{Microscopy evaluation of geometrical melt pool features}

The melt pool depth and width are measured on samples of tracks produced with the optimum condition and the high VED condition. A total of 5 measurements are made for each dimension. The melt pool width is measured by imaging the top surface (Figure 18 (c) and (d)) by means of a Keyence VHX-600 optical microscope. The melt pool depth for the sample produced with high VED (condition 3) is estimated by examining the keyhole porosity depth on a polished cross section by using SEM (Figure 18 (b)). As the sample produced with optimum VED (condition 1) shows no porosity to help determine the melt pool depth, the depth for this condition is estimated by examining the melt pool for a single line scan performed on a cold-rolled Ti-6Al-4V substrate [34]. The substrate is covered with a single layer of powder to mimic processing conditions. This experiment was carried out on a Concept Laser M1 LPBF machine, with similar VED to that for the optimum conditions. The laser power was set at $189 \mathrm{~W}$, the scan speed at $900 \mathrm{~mm} / \mathrm{s}$. The laser beam spot size was $50 \mu \mathrm{m} \emptyset_{1 / e^{2}}$, resulting in a VED of $140 \mathrm{~J} \cdot \mathrm{mm}^{-3}$. A relevant crosssection is shown in Figure 18 (a) revealing a conduction-controlled melt pool, made possible by a difference in microstructure between the substrate and the line scan, readily revealing the melt pool boundaries. A summary of the measurement result is shown in Table 4. For optimum VED, a depth-to-width ratio (aspect ratio) of $\sim 0.85$ is observed, indicating conduction-controlled melting. For the high VED, a depth-to-width ratio (aspect ratio) of $\sim 2.4$ is observed, indicating keyhole mode melting. The melt pool width values from Table 4 are smaller than those determined from the coaxial camera images (Table 3). Future work will focus on the calibration of melt pool grey-level that leads to the same camera melt pool width as the measured track width. 
Table 4. Melt pool dimensions for optimal (condition 1) and high VED (condition 3).

\begin{tabular}{|c|c|c|c|c|c|}
\hline \multicolumn{3}{|c|}{ Optimum VED $\left(\right.$ condition $\left.1, v=1000 \mathrm{~mm} \cdot \mathrm{s}^{-1}\right)$} & \multicolumn{3}{|c|}{ High VED $\left(\right.$ condition $3, v=500 \mathrm{~mm} \cdot \mathrm{s}^{-1}$ ) } \\
\hline Depth / $\mu \mathrm{m}$ & Width / $\mu \mathrm{m}$ & $\begin{array}{c}\text { Depth-to- } \\
\text { width ratio }\end{array}$ & Depth / $\mu \mathrm{m}$ & Width / $\mu \mathrm{m}$ & $\begin{array}{c}\text { Depth-to- } \\
\text { width ratio }\end{array}$ \\
\hline $77 \pm 2$ & $90 \pm 9$ & 0.85 & $198 \pm 9$ & $82 \pm 13$ & 2.4 \\
\hline
\end{tabular}
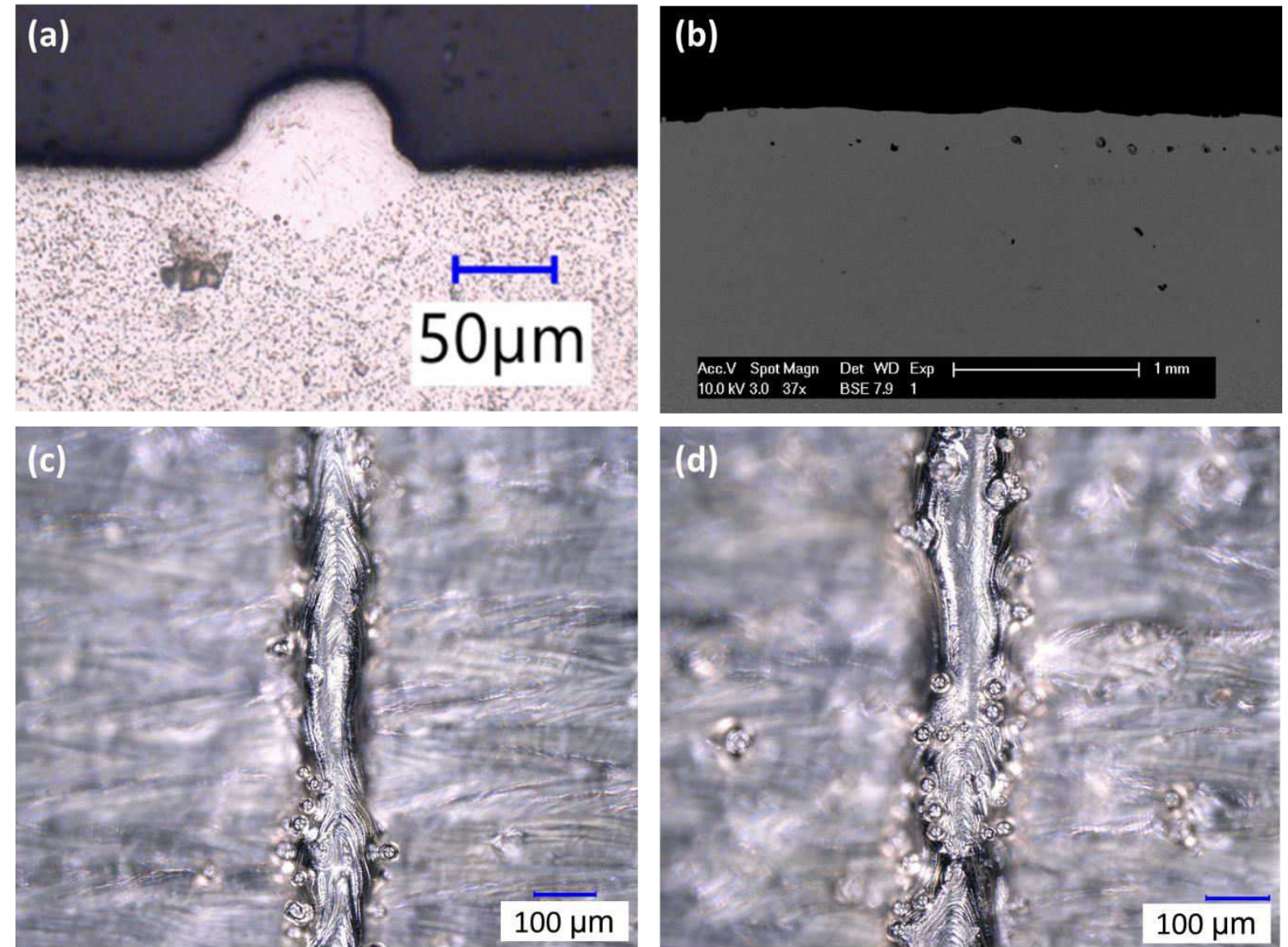

Figure 18. Melt pool geometrical features for Ti-6Al-4V scan track, (a) Melt pool cross-section perpendicular to the line track, produced on a cold-rolled Ti-6Al-4V substrate with a VED of 140 $\mathrm{J} \cdot \mathrm{mm}^{-3}$, similar to condition 1 (Optimum VED); (b) SEM of melt pool cross-section parallel to the line track for the sample of condition 3 (High VED); (c) Top view of the line track with Optimum VED (condition 1); (d) Top view of the line track with High VED (condition 3).

\section{Conclusion}

550 The melt pool intensity and geometry for single tracks melted upon an LPBF substrate are

551 recorded and analysed using coaxial camera melt pool monitoring. Line scans are printed with 552 optimum, low and high volumetric energy density (VED). Extensive keyhole formation was 
observed for the lines printed with high VED at reduced speed. While the average melt pool intensity is the highest for the high VED condition at high speed, the integrated melt pool intensity over print time for a certain line length is the highest for the high VED condition at low speed. These results could indicate that the occurrence of keyhole mode melting is tied with the integrated average of the melt pool radiation, while simultaneously being less dependent on the average measured radiation. Next, image processing and features extraction steps for characterising the melt pool geometry and spatters were applied to the coaxial camera images. Contrary to expectations, no significant difference in the melt pool area, width and length is observed between the optimum and keyhole mode conditions. Also, spatters originating from the melt pool were analysed and counted by using an image processing algorithm for coaxially acquired camera images. For a certain line length, a correlation between the number of keyhole pores and the number of spatter was found, indicating that spatter could be another important inprocess signature of keyhole formation.

CFD simulations provide more insights into the melt pool evolution and keyhole pore formation. From those simulations, it looks like the melt pool depths for the optimum and the keyhole process are very different. The high VED process at low speed shows a very deep melt pool depth possibly leading to keyhole formation and the optimum process only shows a shallow melt pool depth. The increase in melt pool depth over melt pool width ratio for the high VED condition at low speed was confirmed by measuring scan track depth and width of line scan samples.

While this work shows that evaluating the integrated melt pool intensity over print time for a certain line length and detecting spatter could play an important role in assessing the quality of a printed part, building a complete quality assurance system for metal printing will require more research where approaches similar to the one proposed in this paper are needed to get a full understanding of the metal melting process including other types of metal printing defects. The authors will continue their research in this area and extend the proposed approach with more process information to build up a more complete picture of the defect formation process.

\section{Acknowledgement}

This research work was funded by research project "PAM ${ }^{2}$ (Precision Additive Metal Manufacturing)" of the EU Framework Programme for Research and Innovation within Horizon 2020 - Marie Skłodowska-Curie Innovative Training Networks under grant agreement No 721383 and by the agency Flanders Innovation \& Entrepreneurship (VLAIO) through the Flanders Make project MONICON ICON (HBC.2016.0459). The authors acknowledge Prof. Shoufeng Yang (KU Leuven) for initial supervision and discussion of this work.

\section{Statements and Declarations}

\subsection{Funding}

This research work was funded by research project "PAM ${ }^{2}$ (Precision Additive Metal Manufacturing)" of the EU Framework Programme for Research and Innovation within Horizon 2020 - Marie Skłodowska-Curie Innovative Training Networks under grant agreement No 
592721383 and by the agency Flanders Innovation \& Entrepreneurship (VLAIO) through the 593 Flanders Make project MONICON ICON (HBC.2016.0459).

\subsection{Conflicts of interest/Competing interests}

595 The authors have no conflict of interest to declare.

\subsection{Availability of data and material}

597 Not applicable

\subsection{Code availability}

599 Not applicable

600

\subsection{Ethics approval}

601

Approved

602

\subsection{Consent to participate}

603

The authors provide consent to participate.

604

\subsection{Consent for publication}

605 The authors give consent for publication.

\subsection{Authors' contributions}

Aditi Thanki: Conceptualization, investigation, formal analysis, visualization, validation, writing - original draft. Louca Goossens: Conceptualization, formal analysis, writing - review and editing. Agusmian Partogi Ompusunggu: Conceptualization, supervision, investigation, formal analysis, software, writing - original draft. Mohamad Bayat: Investigation, formal analysis, writing - original draft. Abdellatif Bey-Temsamani: Project management, writing original draft. Brecht Van Hooreweder: Supervision, resources, writing - review and editing. Jean-Pierre Kruth: Resources, writing - review and editing. Ann Witvrouw: Conceptualization, supervision, funding acquisition, resources, project management, writing original draft.

\section{7. References}

617 1. Kruth J-P, der Schueren B (1995) Direct production of metallic parts by rapid prototyping.

618 In: Proceedings of the 12th International Congress Laser. pp 123-134

619 2. Meiners W (1999) Direktes Selektives Laser Sintern einkomponentiger metallischer 620 Werkstoffe, RWTH Aachen. Dissertation

621 3. Li C, Pisignano D, Zhao Y, Xue J (2020) Advances in medical applications of additive 622 manufacturing. Engineering 
4. Abe F, Osakada K, Shiomi M, et al (2001) The manufacturing of hard tools from metallic powders by selective laser melting. Journal of Materials Processing Technology 111:210 213. https://doi.org/10.1016/S0924-0136(01)00522-2

5. Leal R, Barreiros FM, Alves L, et al (2017) Additive manufacturing tooling for the automotive industry. The International Journal of Advanced Manufacturing Technology 92:1671-1676

6. Liu R, Wang Z, Sparks T, et al (2017) Aerospace applications of laser additive manufacturing. In: Laser additive manufacturing. Elsevier, pp 351-371

7. Rombouts M, Kruth JP, Froyen L, Mercelis P (2006) Fundamentals of selective laser melting of alloyed steel powders. CIRP Annals - Manufacturing Technology 55:187-192. https://doi.org/10.1016/S0007-8506(07)60395-3

8. Zhang B, Li Y, Bai Q (2017) Defect formation mechanisms in selective laser melting: a review. Chinese Journal of Mechanical Engineering 30:515-527

9. Khairallah SA, Anderson AT, Rubenchik A, King WE (2016) Laser powder-bed fusion additive manufacturing: Physics of complex melt flow and formation mechanisms of pores, spatter, and denudation zones. Acta Materialia 108:36-45

10. Ranjan R, Yang Y, Ayas C, et al (2017) Controlling local overheating in topology optimization for additive manufacturing. In: Proceedings of euspen special interest group meeting: additive manufacturing, Leuven, Belgium

11. King WE, Barth HD, Castillo VM, et al (2014) Observation of keyhole-mode laser melting in laser powder-bed fusion additive manufacturing. Journal of Materials Processing Technology 214:2915-2925

12. Bayat M, Thanki A, Mohanty S, et al (2019) Keyhole-induced porosities in Laser-based Powder Bed Fusion (L-PBF) of Ti6Al4V: High-fidelity modelling and experimental validation. Additive Manufacturing 30:. https://doi.org/10.1016/j.addma.2019.100835

13. Matsunawa A, Kim J-D, Seto N, et al (1998) Dynamics of keyhole and molten pool in laser welding. Journal of laser applications 10:247-254

14. Martin AA, Calta NP, Khairallah SA, et al (2019) Dynamics of pore formation during laser powder bed fusion additive manufacturing. Nature communications 10:1-10

15. Cunningham R, Zhao C, Parab N, et al (2019) Keyhole threshold and morphology in laser melting revealed by ultrahigh-speed x-ray imaging. Science 363:849-852

16. Everton SK, Hirsch M, Stavroulakis PI, et al (2016) Review of in-situ process monitoring and in-situ metrology for metal additive manufacturing. Materials and Design

17. Goossens LR, Van Hooreweder B (2021) A virtual sensing approach for monitoring meltpool dimensions using high speed coaxial imaging during laser powder bed fusion of 
metals. Additive Manufacturing 40:101923

18. Matthews MJ, Guss G, Khairallah SA, et al (2016) Denudation of metal powder layers in laser powder bed fusion processes. Acta Materialia 114:33-42

19. Ly S, Rubenchik AM, Khairallah SA, et al (2017) Metal vapor micro-jet controls material redistribution in laser powder bed fusion additive manufacturing. Scientific reports $7: 1-12$

20. Gunenthiram V, Peyre P, Schneider M, et al (2018) Experimental analysis of spatter generation and melt-pool behavior during the powder bed laser beam melting process. Journal of Materials Processing Technology 251:376-386

21. Repossini G, Laguzza V, Grasso M, Colosimo BM (2017) On the use of spatter signature for in-situ monitoring of Laser Powder Bed Fusion. Additive Manufacturing 16:35-48

22. Thanki A, Goossens L, Mertens R, et al (2019) Study of keyhole-porosities in selective laser melting using X-ray computed tomography. 9th Conference on Industrial Computed Tomography $1-7$

23. Thorlabs (2017) Si Switchable Gain Detector User Guide. 15

24. Mikrotron Gmbh (2016) High-Speed CMOS CameraEoSens®3CL Datasheet. 2

25. Lim JS (1990) Two-dimensional signal and image processing. ph

26. Lee J-S (1980) Digital image enhancement and noise filtering by use of local statistics. IEEE transactions on pattern analysis and machine intelligence 165-168

27. Matas J, Chum O, Urban M, Pajdla T (2004) Robust wide-baseline stereo from maximally stable extremal regions. Image and vision computing 22:761-767

28. Hu M-K (1962) Visual pattern recognition by moment invariants. IRE transactions on information theory 8:179-187

29. Cho J-H, Na S-J (2006) Implementation of real-time multiple reflection and Fresnel absorption of laser beam in keyhole. Journal of Physics D: Applied Physics 39:5372

30. Tan W, Bailey NS, Shin YC (2013) Investigation of keyhole plume and molten pool based on a three-dimensional dynamic model with sharp interface formulation. Journal of Physics D: Applied Physics 46:55501

31. Young ZA, Guo Q, Parab ND, et al (2020) Types of spatter and their features and formation mechanisms in laser powder bed fusion additive manufacturing process. Additive Manufacturing 36:101438

32. Wang D, Wu S, Fu F, et al (2017) Mechanisms and characteristics of spatter generation in SLM processing and its effect on the properties. Materials \& Design 117:121-130

33. Martin AA, Calta NP, Hammons JA, et al (2019) Ultrafast dynamics of laser-metal 
interactions in additive manufacturing alloys captured by in situ X-ray imaging. Materials Today Advances 1:100002

34. Coen V (2020) An analytical melt pool model for Laser-Powder Bed Fusion An experimental approach for the validation and calibration of a model for Ti6Al4V powder. KULeuven 